ApJ, ACCEPTEd (July 7 2013)

Preprint typeset using $\mathrm{LAT}_{\mathrm{E}} \mathrm{X}$ style emulateapj v. 5/2/11

\title{
PLANETS AROUND LOW-MASS STARS (PALMS). III. A YOUNG DUSTY L DWARF COMPANION AT THE DEUTERIUM-BURNING LIMIT*†
}

\author{
Brendan P. Bowler, ${ }^{1}$ Michael C. Liu, ${ }^{1,2}$ Evgenya L. Shkolnik, ${ }^{3}$ Trent J. Dupuy ${ }^{4,5}$ \\ ApJ, Accepted (July 7 2013)
}

\begin{abstract}
We report the discovery of an L-type companion to the young M3.5V star 2MASS J012250932439505 at a projected separation of $1.45^{\prime \prime}(\approx 52 \mathrm{AU})$ as part of our adaptive optics imaging search for extrasolar giant planets around young low-mass stars. 2MASS 0122-2439 B has very red nearinfrared colors similar to the HR 8799 planets and the reddest known young/dusty L dwarfs in the field. Moderate-resolution $(R \approx 3800) \quad 1.5-2.4 \mu \mathrm{m}$ spectroscopy reveals a near-infrared spectral type of L4-L6 and an angular $H$-band shape, confirming its cool temperature and young age. The kinematics of 2MASS 0122-2439 AB are marginally consistent with members of the $\sim 120$ Myr AB Dor young moving group based on the photometric distance to the primary $(36 \pm 4 \mathrm{pc})$ and our radial velocity measurement of 2MASS 0122-2439 A from Keck/HIRES. We adopt the AB Dor group age for the system, but the high energy emission, lack of Li I $\lambda 6707$ absorption, and spectral shape of 2MASS $0122-2439 \mathrm{~B}$ suggest a range of $\sim 10-120 \mathrm{Myr}$ is possible. The age and luminosity of 2MASS 0122-2439 B fall in a strip where "hot-start" evolutionary model mass tracks overlap as a result of deuterium burning. Several known substellar companions also fall in this region (2MASS J01035515 ABb, AB Pic b, $\kappa$ And b, G196-3 B, SDSS 2249+0044 B, LP 261-75 B, HD 203030 B, and HN Peg B), but their dual-valued mass predictions have largely been unrecognized. The implied mass of 2MASS $0122-2439 \mathrm{~B}$ is $\approx 12-13 M_{\text {Jup }}$ or $\approx 22-27 M_{\text {Jup }}$ if it is an AB Dor member, or possibly as low as $11 M_{\text {Jup }}$ if the wider age range is adopted. Evolutionary models predict an effective temperature for 2MASS $0122-2439 \mathrm{~B}$ that corresponds to spectral types near the $\mathrm{L} / \mathrm{T}$ transition $(\approx 1300-1500 \mathrm{~K})$ for field objects. However, we find a mid-L near-infrared spectral type, indicating that 2MASS 01222439 B represents another case of photospheric dust being retained to cooler temperatures at low surface gravities, as seen in the spectra of young (8-30 Myr) planetary companions. Altogether, the low mass, low temperature, and red colors of 2MASS 0122-2439 B make it a bridge between warm planets like $\beta$ Pic b and cool, very dusty ones like HR 8799 bcde.
\end{abstract}

Subject headings: stars: individual (2MASS J01225093-2439505) - stars: low-mass, brown dwarfs

\section{INTRODUCTION}

Adaptive optics imaging is a powerful tool to explore the architecture and demographics of planetary systems beyond 10 AU. A handful of companions below the deuterium-burning limit ( $\sim 13 M_{\text {Jup }}$; Spiegel et al. 2011) have been found, but the origin of these planetary-mass companions is ambiguous since there is growing evidence that objects in the 5-15 $M_{\text {Jup }}$ range can form in multiple ways. The $\beta$ Pic and HR 8799 planets are

bpbowler@ifa.hawaii.edu

${ }^{1}$ Institute for Astronomy, University of Hawai' $i$; 2680 Woodlawn Drive, Honolulu, HI 96822, USA

${ }_{2}^{2}$ Visiting Astronomer at the Infrared Telescope Facility, which is operated by the University of Hawai' $\mathrm{i}$ under Cooperative Agreement no. NNX-08AE38A with the National Aeronautics and Space Administration, Science Mission Directorate, Planetary Astronomy Program.

${ }^{3}$ Lowell Observatory, 1400 W. Mars Hill Road, Flagstaff, AZ 86001

${ }^{4}$ Harvard-Smithsonian Center for Astrophysics, 60 Garden Street, Cambridge, MA 02138

${ }^{5}$ Hubble Fellow

* Some of the data presented herein were obtained at the W.M. Keck Observatory, which is operated as a scientific partnership among the California Institute of Technology, the University of California and the National Aeronautics and Space Administration. The Observatory was made possible by the generous financial support of the W.M. Keck Foundation.

$\dagger$ Based in part on data collected at Subaru Telescope, which is operated by the National Astronomical Observatory of Japan. nested in debris disks and probably originated in massive protoplanetary disks (Marois et al. 2008; Marois et al. 2010; Lagrange et al. 2010), although their exact mode of formation is still unclear (Dodson-Robinson et al. 2009; Nero \& Biorkman 2009; Meru \& Bate 2010). Planetary-mass companions have also been found in distinctly "non-planetary" environments - both orbiting stars at ultra-wide separations (Lafrenière et al. 2008; Ireland et al. 2011; Luhman et al. 2011), and brown dwarfs with large mass ratios (Chauvin et al. 2004; Todorov et al. 2010; Liu et al. 2011) — pointing to an origin more consistent with molecular cloud fragmentation than formation in a disk (Lodato et al. 2005; Bate 2012; Vorobyov 2013). These multiple possibilities make the formation scenario of any individual system difficult to deduce if devoid of environmental clues (Bowler et al. 2011; Bailey et al. 2013).

Direct imaging also enables detailed studies of planetary atmospheres through spectroscopy. Followup observations of the young gas giants orbiting HR 8799 (Bowler et al. 2010; Barman et al. 2011a; Konopacky et al. 2013; Oppenheimer et al. 2013) and the planetary-mass companion 2MASS 1207-3932 b (Skemer et al. 2011; Barman et al. 2011b) confirmed the surprising gravity-dependence of the L-T transition for young L dwarfs (e.g., Metchev \& Hillenbrand 2006; Stephens et al. 2009). In cool, low-gravity atmospheres, 
photospheric dust appears to be retained to lower effective temperatures compared to old (high gravity) brown dwarfs in the field. Marley et al. (2012) provide an intuitive framework for this phenomenon in which the gravity-dependence of cloud particle size and cloud base pressure conspire to produce similar column optical depths in cool, low-gravity photospheres and slightly warmer, high-gravity conditions. This results in spectral types that appear earlier than expected based on evolutionary model-predicted temperatures and spectral type-temperature relations for field objects. However, a detailed understanding of this process is hampered by the small number of young $\mathrm{L}$ and $\mathrm{T}$ dwarfs known.

We are conducting a deep adaptive optics imaging survey of young (10-300 Myr) low-mass (0.2-0.6 $\left.M_{\odot}\right)$ stars to study the outer architectures of $\mathrm{M}$ dwarf planetary systems and to identify giant planets for spectral characterization. In Bowler et al. (2012a) and Bowler et al. (2012b) we presented the discovery of two young ( 120-300 Myr) substellar companions with masses of $32 \pm 6 M_{\text {Jup }}$ and $46 \pm 16 M_{\text {Jup }}$ at separations of $\approx 120 \mathrm{AU}$ and $\approx 4.5 \mathrm{AU}$. Here we present a substellar companion to the active M3.5 star 2MASS J012250932439505 (hereinafter 2MASS 0122-2439). The young age of 2MASS 0122-2439 was first recognized by Riaz et al. (2006) based on its high X-ray luminosity and strong $\mathrm{H} \alpha$ emission $(9.7 \AA)$. In their kinematic analysis of active $\mathrm{M}$ dwarfs, Malo et al. (2013) identified 2MASS 0122-2439 as a likely member of the $\sim 120$ Myr AB Dor young moving group (YMG; Zuckerman et al. 2004). Neither a parallax nor a radial velocity has been published for this otherwise anonymous star. Below we describe the discovery and spectroscopic analysis of a dusty L dwarf companion to 2MASS 0122-2439 with an estimated mass near the deuterium-burning limit.

\section{OBSERVATIONS}

\subsection{Subaru/IRCS Adaptive Optics Imaging}

We first imaged 2MASS 0122-2439 with the Infrared Camera and Spectrograph (IRCS; Kobayashi et al. 2000; Tokunaga et al. 1998) combined with the AO188 adaptive optics system (Hayano et al. 2010) at the Subaru Telescope on 2012 Oct 12 UT. Natural guide star (NGS) adaptive optics was used with 2MASS 0122-2439 A ( $R=13.2 \mathrm{mag}$ ) acting as the wavefront reference. The observations were made with the smallest plate scale (20 mas/pix), resulting in a field of view of $21^{\prime \prime}$ across the $1024 \times 1024$ array. We obtained short (5 exposures $\times 5$ coadds $\times 1.0 \mathrm{~s})$ and long $(2$ exposures $\times 1$ coadd $\times 60 \mathrm{~s}$ ) dithered frames with the $H$ and $K$ filters (Mauna Kea Observatory system; Simons \& Tokunaga 2002; Tokunaga et al. 2002). Seeing was poor $\left(1-2^{\prime \prime}\right)$ and variable throughout the night, resulting in rapid changes in the quality of $\mathrm{AO}$ correction. Dome flats obtained at the start of the night were used to correct for pixel sensitivity variations. A faint $(\Delta \operatorname{mag} \sim 5)$ point source was resolved $\sim 1^{\prime \prime} .5$ from 2 MASS $0122-2439 \mathrm{~A}$, but the AO correction was only only good enough to extract precise astrometry ( $<20$ mas) for a single short-exposure frame.

Astrometry for this image was measured by fitting an analytic model of three elliptical Gaussians to each binary component as described in Liu et al. (2008). As in previous work (Dupuy et al. 2009; Dupuv et al. 2010), we determined the errors in separation and position angle (PA) by fitting simulated data created from the image itself. Images of the primary are successively scaled to the measured flux of the companion and injected at the best-fit separation (70.75 pix), adding random offsets with an rms of 1.0 pixel. We also allowed for random offsets in the flux ratios of our injected companions $(\mathrm{rms}=$ $0.3 \mathrm{mag}$ ), distributing them uniformly in PA but avoiding the $\pm 10 \mathrm{deg} P A$ range where the actual companion is located. The same fitting routine is applied to $10^{2} \mathrm{such}$ simulated companion images.

North alignment and pixel scale was measured using dithered observations of the young 2.! $4 \mathrm{M} / \mathrm{L}$ binary 1RXS J235133.3+312720 AB (Bowler et al. 2012a) acquired on the same night $(10$ exposures $\times 5$ coadds $\times$ $0.5 \mathrm{~s})$ with an identical setup. We measure a plate scale of $20.41 \pm 0.05$ mas pix $^{-1}$ and a North orientation of $+89.03 \pm 0.09$ based on astrometry of the system from Keck/NIRC2 from Bowler et al., in agreement with the IRCS plate scale of 20.53 mas pix ${ }^{-1}$ measured by Currie et al. (2011). Applying our calibration to the 2MASS 0122-2439 AB system gives a separation and PA of $1444 \pm 7$ mas and $216.7 \pm 0.2 \mathrm{deg}$. We did not correct the data for optical distortions since the relative distortion is small compared to our measurement errors for separations of only a few arcseconds.

\subsection{Keck II/NIRC2 Adaptive Optics Imaging}

We obtained follow-up imaging of 2MASS 0122 2439 AB using Keck II/NIRC2 with NGS-AO on the nights of 18 and 19 January 2013 UT, and again on 30 June 2013 UT. The narrow camera mode was used, resulting in a field of view of $10^{\prime \prime} .2 \times 10^{\prime \prime} .2$. Standard dark subtraction, bad pixel correction, and flat fielding was performed as in previous work (e.g., Bowler et al. 2012a). For our astrometry, we adopt the plate scale of 9.952 mas pix $^{-1}$ and angle of +0.252 between the detector columns and celestial north measured by Yelda et al. (2010). Details of the observations are listed in Table 1

2MASS 0122-2439 B was detected in our $H$-, $K$-, and $L^{\prime}$-band data from January 2013 and our $J$ - and $K$ band data from June 2013 (Figure 11), but not in our $J$-band images from January 2013. For the detections, astrometry and relative photometry was extracted either by fitting an analytic model of three elliptical Gaussians to each component or performing aperture photometry with sky subtraction. The standard deviation of multiple measurements is computed to derive the PA and separation uncertainty for each filter. A lower limit for the January $2013 J$-band flux ratio was computed by adding a small image of the primary star scaled to 3 times the background rms at the known position of the companion, and then extracting flux ratios using the same fitting method. The results are listed in Table[1] Although the $K$-band photometry from NIRC2 and IRCS disagree at the 2.2- $\sigma$ level, we note that the companion is wellresolved from the primary in our NIRC2 data, but it sits in a large halo in the IRCS data because of poor seeing that night. The flux ratio from NIRC2 is therefore more reliable than the IRCS measurement, as indicated by their relative uncertainties $(0.04 \mathrm{mag}$ vs $0.24 \mathrm{mag})$. Moreover, our two epochs of $K$-band data from NIRC2 are mutually consistent. 


\subsection{Keck I/OSIRIS Near-Infrared Spectroscopy of 2MASS 0122-2439 B}

We targeted 2MASS 0122-2439 B with the OHSuppressing Infrared Imaging Spectrograph (OSIRIS; Larkin et al. 2006) on 2013 February 4 UT with the Keck I Telescope using NGS-AO. The $H b b$ and $K b b$ filters were used with the 50 mas plate scale, resulting in a lenslet geometry of $16 \times 64$, a field of view of $0.56 \times 2$ ". 24 , and a resolving power $R \equiv \lambda / \Delta \lambda \sim 3800$ (Table2). The long axis of the detector was oriented perpendicular to the binary position angle and nodded along the detector by $\sim 1^{\prime \prime}$ in an ABBA pattern. We obtained a total on-source integration of $35 \mathrm{~min}$ in $K$ and $30 \mathrm{~min}$ in $H$ in $\sim 1^{\prime \prime}$ seeing conditions over an airmass of 1.7-2.3. Immediately after our science observations, we acquired spectra of the A0V star HD 20878 at a similar airmass for telluric correction.

The 2D data were reduced and rectified into wavelength-calibrated $3 \mathrm{D}$ cubes with the OSIRIS Data Reduction Pipeline. A new grating was installed in OSIRIS in December 2012 so we made use of a preliminary set of rectification matrices obtained shortly after our observing run on 2013 February 16 (J. Lyke 2013, private communication). The spectra were extracted using aperture photometry with an aperture radius of 3 spaxels, and were then median-combined after scaling each spectrum to a common level. Measurement uncertainties were derived by computing the standard deviation of the scaled flux level for each spectral channel. Telluric correction was performed using a generalized version of the Spextool reduction package for IRTF/SpeX (Vacca et al. 2003; Cushing et al. 2004). Each band was then flux-calibrated using our photometry measurements in Table 3 .

\subsection{Keck I/HIRES Spectroscopy of 2MASS 0122-2439 A}

We obtained an optical spectrum of 2MASS 0122 2439 A with the High REsolution Echelle Spectrometer (HIRES; Vogt et al. 1994) on the Keck I Telescope on 2011 Dec 28 UT (HJD 2456289.72878786). The HIRESr setting was used with the GG475 filter and a slit width of $0^{\prime \prime} .861$, producing a resolving power of $R=48000$ from $6300-7800 \AA$ and $7850-9200 \AA$ for the green and red chips. The integration time was $90 \mathrm{~s}$, but the $\mathrm{S} / \mathrm{N}$ was somewhat low ( $\sim 20$ per resolution element) as a result of poor dome seeing $\left(\sim 2^{\prime \prime}\right)$. Wavelength calibration was achieved using Th/Ar lamps. Details about the data reduction and spectral extraction can be found in Shkolnik et al. (2009) and Shkolnik et al. (2012).

We measured a radial velocity (RV) for 2MASS 0122 2439 A by cross-correlating the spectra from the red and green chips with the M3.5 RV standard GJ 273 (Nidever et al. 2002) obtained on the same night. We found an RV of $9.5 \pm 1.1 \mathrm{~km} \mathrm{~s}^{-1}$ and $9.7 \pm 0.9 \mathrm{~km}$ $\mathrm{s}^{-1}$ for the red and green chips, respectively. The uncertainties for both chips are the quadrature sum of the following error terms: the rms from 6 (11) spectral orders for 2MASS 0122-2439 A for the red (green) chip $\left(0.9 \mathrm{~km} \mathrm{~s}^{-1}\right.$ and $\left.0.6 \mathrm{~km} \mathrm{~s}^{-1}\right)$, the rms from the standard GJ $273\left(0.4 \mathrm{~km} \mathrm{~s}^{-1}\right)$, and a estimated systematic drift of $\sim 0.5 \mathrm{~km} \mathrm{~s}^{-1}$ during the night. We adopt the mean value of both chips, $9.6 \pm 0.7 \mathrm{~km} \mathrm{~s}^{-1}$.

\subsection{IRTF/SpeX Near-Infrared Spectroscopy}

As a comparison template for our spectrum of 2MASS 0122-2439 B, we present observations of the companion to the young M4.5 star LP 261-75 obtained with IRTF/SpeX in prism mode (Rayner et al. 2003) on 2006 November 19 UT. LP 261-75 B (also known as 2MASS J09510549+3558021 and NLTT 22741 B) is an L6.5 brown dwarf first identified by Kirkpatrick et al. (2000) and found to be comoving with the young $(\sim 100$ 200 Myr) M dwarf LP 261-75 A by Reid \& Walkowicz (2006). A total on-source integration time of 16 min was obtained in an ABBA pattern with the 0.5 slit, yielding a resolving power of $\sim 150$. Arc lamps for wavelength calibration were acquired at the same telescope position. The A0V star HD 89239 was observed at a similar airmass as the science observations for telluric correction. The Spextool reduction package was used to reduce, extract, and telluric-correct the observations (Vacca et al. 2003; Cushing et al. 2004).

\section{RESULTS}

3.1. Distance

Since no parallax is available for 2MASS 0122-2439 A, we estimate its distance photometrically using empirical color-magnitude relations. This requires knowledge of the system age since pre-main sequence isochrones span $\sim 3$ mag in $M_{V}$ (a factor of $\sim 4$ in inferred distance) from 10 Myr to the zero-age main sequence ( $160 \mathrm{Myr}$ for a $0.4 M_{\odot}$ star; Baraffe et al. 1998). As discussed in Section 3.3. the kinematics of 2MASS 0122-2439 AB are marginally consistent with the AB Dor YMG, which has an age similar to the Pleiades (Luhman et al. 2005; Barenfeld et al. 2013). We therefore make use of the Pleiades isochrone to compute a photometric distance for our target. Using the Pleiades membership list from Stauffer et al. (2007) and a cluster distance of $133 \mathrm{pc}$ (Soderblom et al. 2005), we fit a sixth-order polynomial to the cluster sequence in $M_{V}$ vs. $V-K_{S}$. Unresolved binaries will systematically bias this fit to brighter values compared to the single star locus. We therefore shift the distribution $0.15 \mathrm{mag}$ fainter as a compromise between a more populous single star locus and an upper envelope of equal-flux binaries, which will brighten sources by as much as $0.75 \mathrm{mag}$. The resulting fit is $M_{V}=0.15$ $+\sum_{i=0}^{5} c_{i} \times\left(V-K_{S}\right)^{i}$, where $c_{i}=\{2.15195,-2.39272$, $5.95275,-3.25361,0.850172,-0.106731,0.00519175\}$. The rms about the fit is $0.28 \mathrm{mag}$ and it is valid between $V-K_{S}=0.5-6.0$ mag. Applying the relation to 2MASS 0122-2439 A gives $M_{V}=11.5 \pm 0.3 \mathrm{mag}$ and a photometric distance of $36 \pm 4 \mathrm{pc}$. We assume 2MASS 0122-2439 A is single since our NIRC2 images rule out equal-flux binaries down to $\approx 50$ mas, and there is no evidence it is a double-lines spectroscopic binary from our HIRES observations.

As a comparison, the $M_{V}$ vs. $V-J$ relations for mainsequence $M$ dwarfs from Lépine (2005) yield $M_{V}=$ $11.9 \pm 0.2 \mathrm{mag}$ and a main sequence distance estimate of $29_{-8}^{+11}$ pc. Similarly, at the other plausible age extreme $(\approx 10 \mathrm{Myr})$, the $M_{V}$ vs. $(V-K)$ polynomial fit for the $\approx 12$ Myr $\beta$ Pic YMG from Riedel et al. (2011) implies a distance of $58 \pm 2 \mathrm{pc}$. (No rms is given for their fit, so the error only incorporates photometric uncertainties.) We adopt the photometric distance of $36 \pm 4 \mathrm{pc}$ from 
Pleiades isochrones for this work, which agrees with the statistical distance estimate of $33 \pm 1 \mathrm{pc}$ from Malo et al. (2013); however, we note that distances of $\approx 30-60 \mathrm{pc}$ are possible if 2MASS $0122-2439 \mathrm{AB}$ is not a member of the AB Dor group.

\subsection{Common Proper Motion}

The relatively large proper motion of 2MASS 0122 $2439 \mathrm{~A}\left(170 \pm 2.5{\text { mas } \mathrm{yr}^{-1}}^{-1}\right)$ allows us to test whether the candidate companion is comoving based on our IRCS and NIRC2 AO imaging, which span $\approx 8.5$ months in time (Table 1). Figure 2 shows the relative proper and parallactic motion of a stationary background object, adopting a photometric distance of $36 \pm 4 \mathrm{pc}$ and the proper motion from UCAC4 listed in Table 3. Our NIRC2 astrometry is more precise, so we use our second epoch (Jan 2013) of data as the baseline for the background tracks, while our first epoch (Oct 2012) IRCS and third epoch (June 2013) NIRC2 measurements are used to test the background hypothesis. The predicted separation and position angle for a stationary object at epoch 2012.780 is $1^{\prime \prime} .4648 \pm 00^{\prime \prime} 0011$ and $215.92 \pm 0.13^{\circ}$. Our measured separation at that epoch $\left(1^{\prime \prime} .444 \pm 00^{\prime \prime} 007\right)$ is $2.9-\sigma$ from the background model and the measured PA $\left(216.7 \pm 0.2^{\circ}\right)$ differs by $3.3-\sigma$. Likewise, for epoch 2013.495 the predicted background astrometry is $1^{\prime \prime} .487 \pm 0^{\prime \prime} .005$ and $220.68 \pm 0.14^{\circ}$, which is inconsistent with our separation and position angle measurements by $6.1-\sigma$ and $27-\sigma$, respectively. Altogether, our astrometry rule out a background model at the 28$\sigma$ level. Note that if we assume a farther distance of $60 \pm 5 \mathrm{pc}$, which corresponds to a younger primary age of $\approx 10 \mathrm{Myr}$, we can reject the background hypothesis at the $32-\sigma$ level.

Bayesian techniques provides a straightforward way to quantitatively compare the relative merit of the stationary and comoving models (Schwarz 1978, Liddle 2009). For two competing models, the posterior odds $P\left(M_{1} \mid d\right) / P\left(M_{2} \mid d\right)$ for models $M_{1}$ and $M_{2}$ given the data $d$ are equal to the Bayes factor $P\left(d \mid M_{1}\right) / P\left(d \mid M_{2}\right)$ times the prior odds of each model $P\left(M_{1}\right) / P\left(M_{2}\right)$. For simplicity, we assume equal prior odds for both models. Assuming normally-distributed data, the Bayes factor simplifies to the likelihood ratio $e^{-\Delta \chi^{2} / 2}$. Here

$\chi^{2}=\sum_{i=1}^{N-1}\left(\frac{\left(\theta_{\text {meas }, i}-\theta_{\text {pred }, i}\right)^{2}}{\sigma_{\theta, \text { meas }, i}^{2}+\sigma_{\theta, \text { pred }, i}^{2}}+\frac{\left(\rho_{\text {meas }, i}-\rho_{\text {pred }, i}\right)^{2}}{\sigma_{\rho, \text { meas }, i}^{2}+\sigma_{\rho, \text { pred }, i}^{2}}\right)$,

where $\theta, \rho$, and $\sigma$ are the measured and predicted PA, separation, and their associated uncertainties at epoch $i$ for $N$ epochs of astrometry. The reduced $\chi^{2}$ values (2 degrees of freedom) are 1.5 and 390 for the comoving and background scenarios. The Bayes factors for the comoving vs. stationary models correspond to posterior odds of $\log \left(P\left(M_{1}\right) / P\left(M_{2}\right)\right)=168$, indicating a decisive preference for common proper motion.

\subsection{Age and Kinematics}

Multiple lines of evidence point to a young age for the 2MASS 0122-2439 AB system. The primary was detected in the ROSAT (Voges et al. 1999) and the Galaxy Evolution Explorer (GALEX; Martin et al. 2005; Morrissey et al. 2007) all-sky Xray and UV surveys, indicating it has an active corona and chromosphere. High-energy emission is associated with youth for early-M dwarfs (e.g., Shkolnik et al. 2009; Findeisen \& Hillenbrand 2010; Irwin et al. 2011), though it can also be generated from close, tidally-interacting binaries (e.g., Shkolnik et al. 2010). However, our HIRES data show no evidence that 2MASS $0122-2439 \mathrm{~A}$ is an SB2, so the activity is probably an age-related phenomenon. We compute an X-ray luminosity of $\log L_{X}=28.7 \pm 0.2 \mathrm{erg} \mathrm{s}^{-1}$ and a relative X-ray luminosity of $\log \left(L_{X} / L_{\odot}\right)=-$ $3.17 \pm 0.3$ dex using the measured ROSAT count rate $\left(0.0570 \pm 0.0142 \mathrm{cnt} \mathrm{s}^{-1}\right)$, the count rate-to-flux conversion from Fleming et al. (1995), and our distance estimate. The X-ray luminosity of 2MASS $0122-2439 \mathrm{~A}$ is comparable to known YMG members and more active field objects (Preibisch \& Feigelson 2005; Bowler et al. $2012 \mathrm{~b})$. The X-ray luminosity is near the saturation level of $\sim-3$ dex (Riaz et al. 2006).

Young $\mathrm{M}$ dwarfs produce a wealth of chromospheric emission lines, which decrease with age as magnetic dynamo strengths fade (e.g., Delfosse et al. 1998; Wright et al. 2011). As a result, GALEX has become an excellent resource to identify nearby low-mass members of YMGs (Shkolnik et al. 2011; Rodriguez et al. 2011; Shkolnik et al. 2012; Schlieder et al. 2012). The $G A L E X N U V-J$ and $F U V-J$ colors of 2MASS 0122 $2439 \mathrm{~A}$ are $10.59 \pm 0.14 \mathrm{mag}$ and $11.1 \pm 0.3 \mathrm{mag}$, respectively. Compared to YMG members of various ages from Findeisen et al. (2011), 2MASS 0122-2439 A lies redward of the Hyades sequence $(\sim 600 \mathrm{Myr})$ in a region occupied by members of the TW Hya ( $~ 8 \mathrm{Myr})$, $\beta$ Pic ( 12 Myr), Tuc-Hor ( $\sim 30 \mathrm{Myr})$, and AB Dor $(\sim 120 \mathrm{Myr})$ associations. Because of the large scatter in these relations, the high energy activity provides only a coarse age constraint of $\lesssim 500 \mathrm{Myr}$. We measure an $\mathrm{H} \alpha$ $E W$ of $-5.8 \pm 0.5 \AA$ for the primary from our HIRES spectrum, which points to an age less than $\sim 3$ Gyr based on $\mathrm{M}$ dwarf activity lifetimes (West et al. 2008). Note that Riaz et al. (2006) find a value of $-9.7 \AA$, indicating some variability is present 8 .

Malo et al. (2013) identify 2MASS $0122-2439$ as a likely member of the AB Dor YMG (Zuckerman et al. 2004) based on its proper motion, photometry, and sky position. They predict an RV of $15.5 \pm 2.1 \mathrm{~km} \mathrm{~s}^{-1}$, but our measured value of $9.6 \pm 0.7 \mathrm{~km} \mathrm{~s}^{-1}$ differs from this by $2.8 \sigma$. Using the proper motion, RV, and photometric distance of 2 MASS $0122-2439$ A, we compute $U V W$ space velocities and $X Y Z$ galactic positions (Table 3), which are shown in Figure 3 relative to YMG members from Torres et al. (2008). 2MASS 0122-2439 AB agrees with the AB Dor group in $U$ and $V$, but is near the outskirts of known members in $W$. We therefore tentatively adopt the age of the AB Dor YMG for this work ( 120 Myr; Luhman et al. 2005; Barenfeld et al. 2013), but note that other ages are possible if a future parallax measurement shows it does not belong to that moving

8 We note that the Wide-field Infrared Survey Explorer (WISE; Wright et al. 2010) $W 1-W 4$ color of 2MASS 0122-2439 A $(0.8 \pm 0.2 \mathrm{mag})$ may suggest a weak $22 \mu \mathrm{m}$ excess compared to early-M dwarfs in the field, which have colors of $\approx 0.3 \mathrm{mag}$ (Avenhaus et al. 2012). However, the significance of the $22 \mu \mathrm{m}$ detection is only $2.5 \sigma$ so follow-up observations are needed for verification. 
group.

The lack of Li I $\lambda 6708$ absorption in 2MASS 0122 2439 A $(E W<50 \mathrm{~mA})$ from our HIRES spectrum provides a strict lower limit on the age of the system since the $\mathrm{Li}$ depletion boundary is a strong function of temperature and age. Compared to the Li equivalent widths for cool YMG members from Mentuch et al. (2008), 2MASS 0122-2439 A must be older than the TW Hydrae moving group ( $8 \mathrm{Myr})$ based on its spectral type of M3.5. Lithium depletion predictions from evolutionary models can also be used to set a lower age limit on the system. For 2MASS 0122-2439 A, the models of Chabrier et al. (1996) indicate a Li-burning timescale of $\sim 20-30$ Myr. The accretion history of young stars can result in more rapid depletion times (Baraffe \& Chabrier 2010), so 20-30 Myr is effectively the oldest possible lower limit for the system age. However, the spectral shape of 2MASS 0122-2439 B implies an approximate upper age limit of 120 Myr (see Section 3.5.2).

\subsection{Luminosity, Mass, and Effective Temperature}

For the primary 2MASS 0122-2439 A, we derive a luminosity of $\log \left(L / L_{\odot}\right)=-1.72 \pm 0.11$ dex using the $H$-band bolometric correction from Casagrande et al. (2008) and the photometric distance from Section 3.1. At an age of $120 \mathrm{Myr}(10 \mathrm{Myr})$, the luminosity of 2MASS 0122-2439 A implies a mass of $0.40 \pm 0.05 M_{\odot}$ $\left(0.13 \pm 0.02 M_{\odot}\right)$ and an effective temperature of $3530 \pm 50 \mathrm{~K}(3150 \pm 40 \mathrm{~K})$ based on the solar-metallicity evolutionary models of Baraffe et al. (1998).

We calculate the luminosity of 2MASS 0122-2439 B by integrating our flux-calibrated 1.5-2.4 $\mu \mathrm{m}$ spectrum (Section 3.5.2) together with a scaled model spectrum at shorter $(0.001-1.5 \mu \mathrm{m})$ and longer $(2.4-1000 \mu \mathrm{m})$ wavelengths. Based on the mid-L spectral type of 2MASS 0122-2439 B (Section 3.5.2), we use a solar metallicity BT-Settl synthetic spectrum Allard et al. 2010) with an effective temperature of $1700 \mathrm{~K}$ and a loggravity of $4.5 \mathrm{dex}(\mathrm{cgs})$, from which we find a luminosity of $\log L / L_{\odot}=-4.19 \pm 0.10$ dex using our photometric distance. Spectral and photometric (flux calibration) measurement uncertainties together with our photometric distance estimate are taken into account in a Monte Carlo fashion. Note that even though our spectroscopic data only contribute $32 \%$ of the bolometric luminosity, the choice of the model spectrum for the bolometric correction has only a minor influence on the total luminosity since the models are scaled to the flux-calibrated spectra. For example, the $\{1500 \mathrm{~K}, 4.5 \mathrm{dex}\}$ spectrum yields a consistent luminosity of $-4.16 \pm 0.10$ dex.

Figure 4 shows the luminosity and age of 2MASS 0122 2439 B compared to the cloudless (dotted gray tracks) and cloudy (solid gray tracks) "hot start" cooling models of Saumon \& Marley (2008). 2MASS 0122-2439 B sits in a region at the deuterium-burning limit where mass tracks overlap because the onset of deuterium burning is a function of mass (e.g., Burrows et al. 2001; Spiegel et al. 2011). Its position coincides with the 0.012-0.013 $M_{\odot}\left(\sim 12-14 M_{\text {Jup }}\right)$ and $0.022-0.026 M_{\odot}$ ( $\left.\sim 23-27 M_{\text {Jup }}\right)$ cloudy tracks. These mass ranges appear to be more likely for 2MASS 0122-2439 B than the $15-20 M_{\text {Jup }}$ range, which lies at slightly lower luminosities and younger ages. This highlights an important (but often unrecognized) feature for all hot-start evolu- tionary models: in this strip near the deuterium-burning limit, a luminosity and age alone do not coincide with a unique mass. However, as we discuss Section 4 , a detailed spectroscopic comparison of objects at the same location might break this mass degeneracy. Here we have assumed the system belongs to the AB Dor YMG, but if it is not a member then the age may be as low as $\approx 10 \mathrm{Myr}$, in which case the corresponding luminosity and mass of 2MASS $0122-2439 \mathrm{~B}$ are $-3.66 \pm 0.08$ dex and $\approx 11 M_{\text {Jup }}$.

The formation and accretion history of substellar companions can strongly influence the evolution of their luminosity up to ages of $\sim 1$ Gyr (Marley et al. 2007; Fortney et al. 2008; Spiegel \& Burrows 2012). Our hotstart mass estimates, which are based on arbitrarily high initial specific entropies, are in fact lower limits for the actual mass of 2MASS 0122-2439 B if some of the initial gravitational energy is radiated away as accretion luminosity (Marleau \& Cumming 2013). In Figure 5we consider the "cold start" evolutionary models of Mollière \& Mordasini (2012), which are based on a core accretion formation scenario. Although core accretion is unlikely to have created such a massive companion at the present location of 2MASS 0122-2439 B (52 AU in projected separation), it remains possible that dynamical interactions with another close-in companion could have scattered it to a wide orbit (e.g., Veras et al. 2009). Above $\approx 13 M_{\text {Jup }}$, deuterium burning results in a luminosity "bump" which onsets at progressively later ages for lower masses. For 2MASS 0122-2439 B, deuterium burning in the cold start models results in dual-valued masses of $\approx 14 M_{\text {Jup }}$ and $\approx 23 M_{\text {Jup }}$, which are similar to those from the hot start models.

Hot start evolutionary models predict effective temperatures between $\sim 1350-1500 \mathrm{~K}$ based on the luminosity and age of 2MASS 0122-2439 B (Figure 6] ), differing somewhat for the two possible mass regimes. At lower masses of $\sim 13 M_{\mathrm{Jup}}$, temperatures from the Saumon \& Marlev (2008) models range from 1350$1410 \mathrm{~K}$ for the cloudless, cloudy, and hybrid prescriptions. At higher masses of $\sim 24 M_{\mathrm{Jup}}$, the temperatures are between $1450-1500 \mathrm{~K}$.

\subsection{Spectral Properties of 2MASS 0122-2439 B}

\subsubsection{Photometry}

$J-, H$-, and $K$-band photometry for 2MASS 0122 $2439 \mathrm{~B}$ is computed using our measured relative photometry and the apparent magnitudes of 2MASS 0122 2439 A from 2MASS (Skrutskie et al. 2006), converted to the MKO filter system based on the relations from Leggett et al. (2006). The $L^{\prime}$ photometry of 2MASS $0122-2439 \mathrm{~B}$ is derived assuming a $K-L^{\prime}$ color of $0.2 \pm 0.1 \mathrm{mag}$ for the primary, which is the typical value for an M3.5 dwarf (Golimowski et al. 2004). The results are listed in Table 3 .

Figure 7 shows the $H-K$ vs $K-L^{\prime}$ colors of 2MASS 0122-2439 B compared to field M, L, and T dwarfs (Leggett et al. 2010) and the planetary-mass companions HR 8799 bcde, 2MASS $1207-3932 \mathrm{~b}$, and $\beta$ Pic b. The $K-L^{\prime}$ color of 2 MASS $0122-2439$ B is similar to mid$\mathrm{L}$ dwarfs, but the $H-K$ value is redder than most field objects by $\sim 0.3$ mag.

In Figures 8 and 9 we compare the colors and ab- 
solute magnitudes of 2MASS 0122-2439 B (based on its photometric distance) to field $\mathrm{M}, \mathrm{L}$, and $\mathrm{T}$ dwarfs from Dupuy \& Liu (2012) and young substellar companions with parallaxes (see the figure captions for details). 2MASS $0122-2439 \mathrm{~B}$ is similar to mid- to late-L dwarfs in absolute magnitude, but is significantly redder than field objects and most known companions; instead its infrared colors better resemble those of the HR 8799 planets. Compared to young substellar companions, 2MASS 0122-2439 B has the second reddest $J-K$ color after the planetary-mass companion 2MASS J1207-3932 b (Chauvin et al. 2004; Mohanty et al. 2007). The absolute magnitudes and colors of 2MASS 0122-2439 B are remarkably similar to those of 2MASS J03552337+1133437 (hereinafter 2MASS $0355+1133$ ), a young dusty L5 member of the AB Dor moving group (Reid et al. 2008; Cruz et al. 2009; Faherty et al. 2012; Liu et al. 2013). Figures 8 and 9 also emphasize the uniqueness of 2MASS 0122-2439 B; its cool temperature and very red colors make it the only companion currently known with atmospheric properties intermediate between $\beta$ Pic b and the HR 8799 planets.

\subsubsection{Spectroscopy}

In Figure 10 we compare our flux-calibrated spectrum of 2MASS 0122-2439 B to old L dwarfs in the field (top panel) and those exhibiting signs of youth (bottom panel). The $H$-band shape of 2MASS 0122 $2439 \mathrm{~B}$ is significantly more angular than field objects, a prominent signature of low surface gravity (e.g., Lucas et al. 2001; Allers et al. 2007; Allers \& Liu 2013) caused by diminished steam and collision-induced $\mathrm{H}_{2}$ absorption (Barman et al. 2011a). 2MASS 0122 2439 B appears significantly later than the young field L0 object 2MASS J01415823-4633574 (Kirkpatrick et al. 2006) and the L3 companion to the AB Dor member CD352722 (Wahhaj et al. 2011), but somewhat earlier than the young L6.5 brown dwarf 2MASSW J2244316+204343 (Dahn et al. 2002; McLean et al. 2003; Kirkpatrick et al. 2008). Compared to other young L dwarfs in Figure 11. 2MASS $0122-2439 \mathrm{~B}$ has a similar $H$ band shape to the 5-10 Myr L4 companion 1RXS 160929.1-210524 b (Lafrenière et al. 2010), the $\sim 20-100$ Myr L3 companion G196-3 B (Rebolo et al. 1998; Kirkpatrick et al. 2008; Osorio et al. 2010), and the young field L3 dwarf 2MASS J1615425+495321 (Cruz et al. 2007; Kirkpatrick et al. 2008; Geißler et al. 2011; Allers \& Liu 2013). We note that despite its similar colors to the young ( $120 \mathrm{Myr})$ L5 object 2MASS $0355+1133$, 2MASS 0122-2439 B exhibits a more angular $H$-band shape and deeper CO absorption bands, pointing to a younger age and/or lower temperature. Unfortunately, we cannot use the new index-based spectral classification scheme of Allers \& Liu (2013) since our wavelength coverage is not wide enough for their $H$-band index. Altogether, we adopt a NIR spectral type of L4-L6 based on these relative comparisons, although spectroscopy including the $J$ band is needed for a firmer classification.

\subsection{Properties of LP 261-75 B}

LP 261-75 B is an L6.5 (optical type) common proper motion companion to the active M4.5 star LP 261-75 (Reid \& Walkowicz 2006). Reid \& Walkowicz infer an age of 100-200 Myr based on the primary star's coronal activity. At this age, evolutionary models predict a mass of $\approx 20 M_{\text {Jup }}$ for the companion, making it a valuable benchmark system at an intermediate age to compare with 2MASS 0122-2439 B. Here we discuss the properties of LP 261-75 B based on our SpeX prism spectrum (Section 2.5) and a new parallactic distance to the system.

We calculate the luminosity of the young companion LP 261-75 B using the same method for 2MASS 01222439 B in Section 3.4. Our SpeX prism spectrum is first flux-calibrated to the 2MASS $H$-band magnitude $(15.90 \pm 0.14 \mathrm{mag})$. Using a solar metallicity $\left\{T_{\text {eff }}=1500 \mathrm{~K}, \log g=4.5 \mathrm{dex}\right\}$ BT-Settl spectrum for a bolometric correction together with the parallactic distance of $32.95_{-2.40}^{+2.80}$ pc (Vrba et al. 2014, in preparation; updated from Vrba et al. 2004), we measure a bolometric luminosity of $-4.43 \pm 0.09$ dex. Note that our $0.8-2.45 \mu \mathrm{m}$ spectrum represents $51 \%$ of the bolometric flux, but the model choice for the bolometric correction only has a modest influence on the bolometric luminosity ( $\sim 0.03$ dex $)$. As shown in Figure 4, the luminosity and age (100-200 Myr; Reid \& Walkowicz 2006) of LP 261$75 \mathrm{~B}$ is consistent with $\sim 13 M_{\mathrm{Jup}}$ and $\sim 22 M_{\mathrm{Jup}}$ based on a hot start formation.

With our new prism spectrum we also compute precise colors for LP 261-75 B since the detections from 2MASS are near the survey limit. Synthetic photometry based on Monte Carlo realizations of our spectrum yields the following colors: $(J-H)_{\mathrm{MKO}}=1.081 \pm 0.003 \mathrm{mag}$, $(H-K)_{\mathrm{MKO}}=0.828 \pm 0.002 \mathrm{mag},(J-K)_{\mathrm{MKO}}=$ $1.908 \pm 0.002 \mathrm{mag},(J-H)_{2 \mathrm{MASS}}=1.239 \pm 0.002 \mathrm{mag}$, $\left(H-K_{S}\right)_{2 \mathrm{MASS}}=0.779 \pm 0.002 \mathrm{mag},\left(J-K_{S}\right)_{2 \mathrm{MASS}}=$ $2.018 \pm 0.002 \mathrm{mag}$. Finally, we compute a NIR spectral type using the classification scheme from Geballe et al. (2002). The $1.5 \mu \mathrm{m} \mathrm{H}_{2} \mathrm{O}$ and $2.2 \mu \mathrm{m} \mathrm{CH}_{4}$ indices yield $\mathrm{L} 3.0 \pm 1.0$ and $\mathrm{L} 6 \pm 1.0$ classifications; the weighted average of these is L4.5 \pm 0.7 . In a large analysis of young brown dwarf near-infrared spectra, Allers \& Liu 2013 found that several index-based classification schemes are relatively insensitive to gravity. Among these is the " $\mathrm{H}_{2} \mathrm{OD}$ " index from McLean et al. (2003), which is applicable for L0-L8 types. Applying the updated index widths from Allers \& Liu yields L4.5 \pm 0.8 , where the uncertainty incorporates measurement errors and the rms from the relation. The gravity score for LP 261-75 B from the Allers \& Liu scheme is 2, indicating a high gravity ("FLD-G") comparable to old field objects. Altogether we adopt a near-infrared spectral type of L4.5 \pm 1.0 for LP 261-75 B.

\section{DISCUSSION}

The luminosity and age of 2MASS 0122-2439 B reside in a region where evolutionary models have dual-valued mass predictions. Comparing other young $(<1$ Gyr) substellar companions to the cooling models in Figure 4 shows that several known objects also fall in or very close to this region: 2MASS J01033563-5515561 ABb (Delorme et al. 2013), AB Pic b (Chauvin et al. 2005), $\kappa$ And b (Carson et al. 2013), G196-3 B (Rebolo et al. 1998), SDSS J224953.47+004404.6 B (Allers et al. 2010), LP261-75 B (Reid \& Walkowicz 2006), HD 203030 B (Metchev \& Hillenbrand 2006), and HN Peg B (Luhman et al. 2007). For these objects, a luminosity 
and age do not translate into a unique mass prediction, instead being consistent with both $\approx 12-14 M_{\text {Jup }}$ and $\approx 20-26 M_{\text {Jup }}$ mass tracks.

One possible way to break this degeneracy may be by comparing the spectra of objects with very similar luminosities and ages but different masses. A lower mass object will have a lower surface gravity which would be reflected in gravity-sensitive features in its spectrum. At the age and luminosity 2MASS 0122 2439 B, the Saumon \& Marley (2008) evolutionary models predict differences of $\approx 0.36$ dex in $\log g$ between the lower (deuterium-burning) and higher (post-deuteriumburning) mass regimes. LP 261-75 B has a similar age and luminosity, but our spectra of the two companions are substantially different from each other (Figure 12). The more angular $H$ band of 2MASS 0122-2439 B suggests a lower gravity than LP 261-75 B, perhaps indicating that 2MASS 0122-2439 B belongs to the lowermass ( $\approx 13 M_{\text {Jup }}$ ) set of model tracks while LP $261-75 \mathrm{~B}$ belongs to the higher-mass $\left(\approx 25 M_{\text {Jup }}\right)$ set. However, uncertainties in the ages of these systems are large, so this might instead point to a younger relative age for 2MASS 0122-2439 B. SDSS 2244+0044 B also has a similar age and luminosity as 2MASS 0122-2439 B and may also be burning deuterium (Allers et al. 2010), but resolved spectroscopy of this binary has only been obtained in $K$ band, which is less sensitive to differences in gravity than $J$ and $H$ bands so we cannot use this as a comparison point. Similarly, while the absolute magnitude and colors of 2MASS 0122-2439 B are consistent with 2 MASS $0355+1133$, the $H$-band shape is somewhat narrower and the $2.3 \mu \mathrm{m} \mathrm{CO}$ absorption is significantly stronger in 2MASS 0122-2439 B. As discussed in Allers \& Liu (2013), this bolsters the notion there may be a diversity of spectral shapes at a given luminosity and age among young dusty L dwarfs; more discoveries are needed to map the influence of gravity, clouds, and temperature in this regime.

For field objects, the effective temperature for 2MASS 0122-2439 B from evolutionary models (1350$1500 \mathrm{~K}$, Section 3.4 corresponds to spectral types spanning the $\mathrm{L} / \mathrm{T}$ transition $(\sim \mathrm{L} 7-\mathrm{T} 2)$. However, we find that 2MASS 0122-2439 B better matches young mid-L spectral types. A similar disagreement between evolutionary model predictions and the temperatures estimated from spectral classification has been noted for a handful of low-temperature substellar objects: HD 203030 B (Metchev \& Hillenbrand 2006), HN Peg B (Luhman et al. 2007), 2MASS 1207-3932 b (Skemer et al. 2011; Barman et al. 2011b), and the HR 8799 planets (Bowler et al. 2010; Currie et al. 2011; Barman et al. 2011b). This phenomenon is illustrated in Figure 13. which shows the observed NIR spectral types of old field objects and young companions compared to the temperatures predicted by the Burrows et al. (1997) evolutionary models based on their ages and luminosities. For a given predicted effective temperature, low gravity objects tend to have earlier spectral types than the field population. This suggests that the spectral type-effective temperature sequence is also a function of gravity (age), becoming most apparent for young objects near the $\mathrm{L} / \mathrm{T}$ transition when dust begins to settle below the photosphere at a fixed effective temperature of

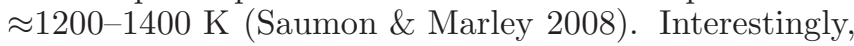

several other young companions (1RXS 2351+3127 B, AB Pic b, G196-3 B, and $\beta$ Pic b) may also exhibit this phenomenon at higher effective temperatures, but more precise spectral types are needed to confirm this. For 2MASS 0122-2439 B, a parallax measurement together with broader NIR spectroscopic coverage will enable a more detailed analysis of the gravity-dependent $\mathrm{L} / \mathrm{T}$ transition.

We thank Fred Vrba and the USNO group for sharing their parallax to LP 261-75 B; Katelyn Allers, Zahed Wahhaj, Davy Kirkpatrick, David Lafrenière, and Jennifer Patience for the low gravity spectra used in this work; Yosuke Minowa for assistance with the IRCS observations; Eric Nielsen for his compilation of YMG members; and Katelyn Allers for assistance with the LP 261-75 B observations. It is a pleasure to thank the Keck support astronomers and operating assistants who helped make this work possible: Joel Aycock, Randy Campbell, Marc Kassis, Jim Lyke, Terry Stickel, and Hien Tran. B.P.B. and M.C.L. have been supported by NASA grant NNX11AC31G and NSF grant AST0909222. E.S. has been supported by NASA/GALEX grant NNX12AC18G. We utilized data products from the Two Micron All Sky Survey, which is a joint project of the University of Massachusetts and the Infrared Processing and Analysis Center/California Institute of Technology, funded by the National Aeronautics and Space Administration and the National Science Foundation. This publication makes use of data products from the Widefield Infrared Survey Explorer, which is a joint project of the University of California, Los Angeles, and the Jet Propulsion Laboratory/California Institute of Technology, funded by the National Aeronautics and Space Administration (NASA). NASA's Astrophysics Data System Bibliographic Services, the VizieR catalogue access tool, and the SIMBAD database operated at CDS, Strasbourg, France, were invaluable resources for this work. Finally, mahalo nui loa to the kama'āina of Hawai'i for their support of Keck and the Mauna Kea observatories. We are grateful to conduct observations from this mountain. 


\section{REFERENCES}

Allard, F., Homeier, D., \& Freytag, B. 2010, arXiv/astro-ph:1011.5405

Allers, K. N., et al. 2007, ApJ, 657, 511

Allers, K. N., \& Liu, M. C. 2013, arXiv/astro-ph:1305.4418

Allers, K. N., Liu, M. C., Dupuy, T. J., \& Cushing, M. C. 2010 , ApJ, 715, 561

Avenhaus, H., Schmid, H. M., \& Meyer, M. R. 2012, Astronomy \& Astrophysics, 548, A105

Bailey, V., et al. 2013, arXiv, astro-ph.SR

Baraffe, I., \& Chabrier, G. 2010, A\&A, 521, A44

Baraffe, I., Chabrier, G., Allard, F., \& Hauschildt, P. H. 1998, A\&A, 337, 403

Barenfeld, S. A., Bubar, E. J., Mamajek, E. E., \& Young, P. A. 2013, ApJ, 766, 6

Barman, T. S., Macintosh, B., Konopacky, Q. M., \& Marois, C. 2011a, ApJ, 733, 65

-. 2011b, ApJ, 735, L39

Bate, M. R. 2012, MNRAS, 419, 3115

Bonnefoy, M., et al. 2013, arXiv/astro-ph:1302.1160

Bonnefoy, M., Chauvin, G., Rojo, P., Allard, F., Lagrange, A.-M. Homeier, D., Dumas, C., \& Beuzit, J.-L. 2010, A\&A, 512, A52

Bowler, B. P., Liu, M. C., Dupuy, T. J., \& Cushing, M. C. 2010, ApJ, 723, 850

Bowler, B. P., Liu, M. C., Kraus, A. L., Mann, A. W., \& Ireland, M. J. 2011, ApJ, 743, 148

Bowler, B. P., Liu, M. C., Shkolnik, E. L., Dupuy, T. J., Cieza, L. A., Kraus, A. L., \& Tamura, M. 2012a, ApJ, 753, 142

Bowler, B. P., Liu, M. C., Shkolnik, E. L., \& Tamura, M. 2012b, ApJ, 756, 69

Burgasser, A. J., et al. 2010, ApJ, 725, 1405

Burrows, A., Hubbard, W. B., Lunine, J. I., \& Liebert, J. 2001, Reviews of Modern Physics, 73, 719

Burrows, A., et al. 1997, ApJ, 491, 856

Carson, J., et al. 2013, ApJ, 763, L32

Casagrande, L., Flynn, C., \& Bessell, M. 2008, MNRAS, 389, 585

Chabrier, G., Baraffe, I., \& Plez, B. 1996, Astrophysical Journal Letters v.459, 459, L91

Chauvin, G., Lagrange, A.-M., Dumas, C., Zuckerman, B., Mouillet, D., Song, I., Beuzit, J.-L., \& Lowrance, P. 2004, A\&A, 425, L29

Chauvin, G., et al. 2005, A\&A, 438, L29

Cruz, K. L., Kirkpatrick, J. D., \& Burgasser, A. J. 2009, AJ, 137, 3345

Cruz, K. L., et al. 2007, AJ, 133, 439

Currie, T., et al. 2011, ApJ, 729, 128

Cushing, M. C., Rayner, J. T., \& Vacca, W. D. 2005, ApJ, 623, 1115

Cushing, M. C., Vacca, W. D., \& Rayner, J. T. 2004, PASP, 116, 362

Cutri, R. M., et al. 2012, VizieR On-line Data Catalog, 2311, 0

Dahn, C. C., et al. 2002, AJ, 124, 1170

Delfosse, X., Forveille, T., Perrier, C., \& Mayor, M. 1998, A\&A, 331,581

Delorme, P., et al. 2013, Astronomy \& Astrophysics, 553, L5

Dodson-Robinson, S. E., Veras, D., Ford, E. B., \& Beichman, C. A. 2009, ApJ, 707, 79

Dupuy, T. J., \& Liu, M. C. 2012, ApJSS, 201, 19

Dupuy, T. J., Liu, M. C., Bowler, B. P., Cushing, M. C., Helling, C., Witte, S., \& Hauschildt, P. 2010, ApJ, 721, 1725

Dupuy, T. J., Liu, M. C., \& Ireland, M. J. 2009, ApJ, 699, 168

Faherty, J. K., Rice, E. L., Cruz, K. L., Mamajek, E. E., \& Núñez, A. 2012, AJ, 145, 2

Findeisen, K., \& Hillenbrand, L. 2010, AJ, 139, 1338

Findeisen, K., Hillenbrand, L., \& Soderblom, D. 2011, AJ, 142, 23

Fleming, T. A., Molendi, S., Maccacaro, T., \& Wolter, A. 1995, Astrophysical Journal Supplement v.99, 99, 701

Fortney, J. J., Marley, M. S., Saumon, D., \& Lodders, K. 2008, ApJ, 683, 1104

Geballe, T. R., et al. 2002, ApJ, 564, 466

Geißler, K., Metchev, S., Kirkpatrick, J. D., Berriman, G. B., \& Looper, D. 2011, ApJ, 732, 56

Golimowski, D. A., et al. 2004, AJ, 127, 3516

Hayano, Y., et al. 2010, Adaptive Optics Systems II. Edited by Ellerbroek, 7736, 21
Ireland, M. J., Kraus, A., Martinache, F., Law, N., \& Hillenbrand, L. A. 2011, ApJ, 726, 113

Irwin, J., Berta, Z. K., Burke, C. J., Charbonneau, D., Nutzman, P., West, A. A., \& Falco, E. E. 2011, ApJ, 727, 56

Kirkpatrick, J. D., Barman, T. S., Burgasser, A. J., McGovern, M. R., McLean, I. S., Tinney, C. G., \& Lowrance, P. J. 2006, ApJ, 639, 1120

Kirkpatrick, J. D., et al. 2008, ApJ, 689, 1295

Kirkpatrick, J. D., Dahn, C. C., Monet, D. G., Reid, I. N., Gizis, J. E., Liebert, J., \& Burgasser, A. J. 2001, AJ, 121, 3235

Kirkpatrick, J. D., et al. 2000, AJ, 120, 447

Kobayashi, N., et al. 2000, Proc. SPIE Vol. 4008, 4008, 1056

Konopacky, Q. M., Barman, T. S., Macintosh, B. A., \& Marois, C. 2013, Science, 339, 1398

Lafrenière, D., Jayawardhana, R., \& van Kerkwijk, M. H. 2008, ApJ, 689, L153

-. 2010, ApJ, 719, 497

Lagrange, A.-M., et al. 2010, Science, 329, 57

Larkin, J., et al. 2006, Ground-based and Airborne Instrumentation for Astronomy. Edited by McLean, 6269, 42 Leggett, S. K., et al. 2010, ApJ, 710, 1627

-. 2006, MNRAS, 373, 781

Lépine, S. 2005, AJ, 130, 1680

Liddle, A. R. 2009, Annual Review of Nuclear and Particle Science, 59, 95

Liu, M. C., et al. 2011, ApJ, 740, 108

Liu, M. C., Dupuy, T. J., \& Allers, K. N. 2013, Astronomische Nachrichten, 334,85

Liu, M. C., Dupuy, T. J., \& Ireland, M. J. 2008, ApJ, 689, 436

Liu, M. C., Dupuy, T. J., \& Leggett, S. K. 2010, ApJ, 722, 311

Lodato, G., Delgado-Donate, E., \& Clarke, C. J. 2005, Monthly Notices RAS Letters, 364, L91

Lucas, P. W., Roche, P. F., Allard, F., \& Hauschildt, P. H. 2001, MNRAS, 326, 695

Luhman, K. L., Burgasser, A. J., \& Bochanski, J. J. 2011, ApJ, 730, L9

Luhman, K. L., et al. 2007, ApJ, 654, 570

Luhman, K. L., Stauffer, J. R., \& Mamajek, E. E. 2005, ApJ, 628, L69

Malo, L., Doyon, R., Lafrenière, D., Artigau, É., Gagné, J., Baron, F., \& Riedel, A. 2013, ApJ, 762, 88

Marleau, G. D., \& Cumming, A. 2013, arXiv, astro-ph.EP

Marley, M. S., Fortney, J. J., Hubickyj, O., Bodenheimer, P., \& Lissauer, J. J. 2007, ApJ, 655, 541

Marley, M. S., Saumon, D., Cushing, M., Ackerman, A. S.,

Fortney, J. J., \& Freedman, R. 2012, ApJ, 754, 135

Marois, C., Macintosh, B., Barman, T., Zuckerman, B., Song, I., Patience, J., Lafreniere, D., \& Doyon, R. 2008, Science, 322, 1348

Marois, C., Zuckerman, B., Konopacky, Q. M., Macintosh, B., \& Barman, T. 2010, Nature, 468, 1080

Martin, D. C., et al. 2005, ApJ, 619, L1

McLean, I. S., McGovern, M. R., Burgasser, A. J., Kirkpatrick, J. D., Prato, L., \& Kim, S. S. 2003, ApJ, 596, 561

Mentuch, E., Brandeker, A., van Kerkwijk, M. H., Jayawardhana, R., \& Hauschildt, P. H. 2008, ApJ, 689, 1127

Meru, F., \& Bate, M. R. 2010, MNRAS, 406, 2279

Metchev, S. A., \& Hillenbrand, L. A. 2004, ApJ, 617, 1330

—. 2006, ApJ, 651, 1166

Mohanty, S., Jayawardhana, R., Huélamo, N., \& Mamajek, E. 2007, ApJ, 657, 1064

Mollière, P., \& Mordasini, C. 2012, Astronomy \& Astrophysics, 547, A105

Monet, D. G., et al. 2003, AJ, 125, 984

Morrissey, P., et al. 2007, ApJSS, 173, 682

Nero, D., \& Bjorkman, J. E. 2009, ApJL, 702, L163

Nidever, D. L., Marcy, G. W., Butler, R. P., Fischer, D. A., \& Vogt, S. S. 2002, ApJSS, 141, 503

Oppenheimer, B. R., et al. 2013, arXiv, astro-ph.EP

Osorio, M. R. Z., Rebolo, R., Bihain, G., Béjar, V. J. S., Caballero, J. A., \& Álvarez, C. 2010, ApJ, 715, 1408

Patience, J., King, R. R., Rosa, R. J. D., \& Marois, C. 2010 , A\&A, 517, A76

Preibisch, T., \& Feigelson, E. D. 2005, ApJSS, 160, 390 
Rayner, J. T., Toomey, D. W., Onaka, P. M., Denault, A. J., Stahlberger, W. E., Vacca, W. D., Cushing, M. C., \& Wang, S. 2003, PASP, 115, 362

Rebolo, R., Osorio, M. R. Z., Madruga, S., Bejar, V. J. S., Arribas, S., \& Licandro, J. 1998, Science, 282, 1309

Reid, I. N., Cruz, K. L., Kirkpatrick, J. D., Allen, P. R., Mungall, F., Liebert, J., Lowrance, P., \& Sweet, A. 2008, AJ, 136, 1290

Reid, I. N., \& Walkowicz, L. M. 2006, PASP, 118, 671

Riaz, B., Gizis, J. E., \& Harvin, J. 2006, AJ, 132, 866

Riedel, A. R., Murphy, S. J., Henry, T. J., Melis, C., Jao, W.-C., \& Subasavage, J. P. 2011, AJ, 142, 104

Rodriguez, D. R., Bessell, M. S., Zuckerman, B., \& Kastner, J. H. 2011, ApJ, 727, 62

Saumon, D., \& Marley, M. S. 2008, ApJ, 689, 1327

Schlieder, J. E., Lépine, S., \& Simon, M. 2012, AJ, 143, 80

Schwarz, G. 1978, The Annals of Statistics, 461

Shkolnik, E., Liu, M. C., \& Reid, I. N. 2009, ApJ, 699, 649

Shkolnik, E. L., Anglada-Escudé, G., Liu, M. C., Bowler, B. P., Weinberger, A. J., Boss, A. P., Reid, I. N., \& Tamura, M. 2012, ApJ, 758, 56

Shkolnik, E. L., Hebb, L., Liu, M. C., Reid, I. N., \& Cameron, A. C. 2010, ApJ, 716, 1522

Shkolnik, E. L., Liu, M. C., Reid, I. N., Dupuy, T., \& Weinberger, A. J. 2011, ApJ, 727, 6

Simons, D. A., \& Tokunaga, A. 2002, PASP, 114, 169

Skemer, A. J., Close, L. M., Szúcs, L., Apai, D., Pascucci, I., \& Biller, B. A. 2011, ApJ, 732, 107

Skemer, A. J., et al. 2012, ApJ, 753, 14

Skrutskie, M. F., et al. 2006, AJ, 131, 1163
Soderblom, D. R., Nelan, E., Benedict, G. F., McArthur, B., Ramirez, I., Spiesman, W., \& Jones, B. F. 2005, AJ, 129, 1616 Spiegel, D. S., \& Burrows, A. 2012, ApJ, 745, 174

Spiegel, D. S., Burrows, A., \& Milsom, J. A. 2011, ApJ, 727, 57

Stauffer, J. R., et al. 2007, ApJSS, 172, 663

Stephens, D. C., et al. 2009, ApJ, 702, 154

Todorov, K., Luhman, K. L., \& Mcleod, K. K. 2010, ApJ, 714, L84

Tokunaga, A. T., et al. 1998, Proc. SPIE Vol. 3354, 3354, 512

Tokunaga, A. T., Simons, D. A., \& Vacca, W. D. 2002, PASP, 114,180

Torres, C. A. O., Quast, G. R., Melo, C. H. F., \& Sterzik, M. F. 2008, Handbook of Star Forming Regions, 757

Vacca, W. D., Cushing, M. C., \& Rayner, J. T. 2003, PASP, 115, 389

Veras, D., Crepp, J. R., \& Ford, E. B. 2009, ApJ, 696, 1600

Voges, W., et al. 1999, A\&A, 349, 389

Vogt, S. S., et al. 1994, Proc. SPIE, 2198, 362

Vorobyov, E. I. 2013, arXiv, astro-ph.EP

Vrba, F. J., et al. 2004, AJ, 127, 2948

Wahhaj, Z., et al. 2011, ApJ, 729, 139

West, A. A., Hawley, S. L., Bochanski, J. J., Covey, K. R., Reid, I. N., Dhital, S., Hilton, E. J., \& Masuda, M. 2008, AJ, 135, 785

Wright, E. L., et al. 2010, AJ, 140, 1868

Wright, N. J., Drake, J. J., Mamajek, E. E., \& Henry, G. W. 2011, ApJ, 743, 48

Yelda, S., Lu, J. R., Ghez, A. M., Clarkson, W., Anderson, J., Do, T., \& Matthews, K. 2010, ApJ, 725, 331

Zacharias, N., Finch, C. T., Girard, T. M., Henden, A., Bartlett,

J. L., Monet, D. G., \& Zacharias, M. I. 2013, AJ, 145, 44

Zuckerman, B., Song, I., \& Bessell, M. S. 2004, ApJ, 613, L65 
TABLE 1

Adaptive Optics Imaging OF 2MASS J01225093-2439505 AB

\begin{tabular}{cccccccc}
\hline \hline $\begin{array}{c}\text { Date } \\
(\mathrm{UT})\end{array}$ & Tel/Inst & Filter & $\begin{array}{c}N \times \text { Coadds } \times \\
\text { Exp. Time }(\mathrm{s})\end{array}$ & $\begin{array}{c}\text { FWHM } \\
(\mathrm{mas})\end{array}$ & $\begin{array}{c}\text { Separation } \\
(")\end{array}$ & $\begin{array}{c}\text { PA } \\
\left(^{\circ}\right)\end{array}$ & $\Delta$ mag \\
\hline 2012 Oct 12 & Subaru/IRCS & $K$ & $1 \times 5 \times 1.0$ & 128 & $1.444 \pm 0.007$ & $216.7 \pm 0.2$ & $4.82 \pm 0.24$ \\
2013 Jan 18 & Keck-II/NIRC2 & $K$ & $5 \times 50 \times 0.3$ & $55.1 \pm 1.4$ & $1.4486 \pm 0.0006$ & $216.64 \pm 0.08$ & $5.36 \pm 0.04$ \\
2013 Jan 18 & Keck-II/NIRC2 & $H$ & $3 \times 50 \times 0.3$ & $51 \pm 2$ & $1.4495 \pm 0.0015$ & $216.59 \pm 0.08$ & $6.18 \pm 0.04$ \\
2013 Jan 18 & Keck-II/NIRC2 & $J$ & $3 \times 50 \times 0.3$ & $50 \pm 4$ & $\ldots$ & $\cdots$ & $>5.8$ \\
2013 Jan 19 & Keck-II/NIRC2 & $L^{\prime}$ & $4 \times 100 \times 0.5$ & $101 \pm 7$ & $1.452 \pm 0.005$ & $216.6 \pm 0.4$ & $4.19 \pm 0.03$ \\
2013 Jun 30 & Keck-II/NIRC2 & $K$ & $4 \times 20 \times 0.3$ & $80 \pm 14$ & $1.448 \pm 0.004$ & $216.47 \pm 0.07$ & $5.35 \pm 0.04$ \\
2013 Jun 30 & Keck-II/NIRC2 & $J$ & $5 \times 10 \times 2.0$ & $120 \pm 20$ & $1.433 \pm 0.010$ & $216.9 \pm 0.4$ & $6.79 \pm 0.14$ \\
\hline
\end{tabular}

Note. - NIRC2 FWHM measurements are computed using the IDL routine NIRC2STREHL made available by Keck Observatory. The IRCS FWHM measurement is for a single image.

TABLE 2

SPeCtroscopy of 2MASS J01225093-2439505 AB

\begin{tabular}{|c|c|c|c|c|}
\hline $\begin{array}{l}\text { Date } \\
\text { (UT) }\end{array}$ & Target & $\begin{array}{l}\text { Telescope/ } \\
\text { Instrument }\end{array}$ & Filter & $N \times \underset{(\mathrm{s})}{\text { Exp. Time }}$ \\
\hline 2013 Feb 02 & 2MASS 0122-2439 B & Keck-I/OSIRIS & $K b b$ & $7 \times 300$ \\
\hline 2013 Feb 02 & 2MASS 0122-2439 B & Keck-I/OSIRIS & $H b b$ & $6 \times 300$ \\
\hline 2012 Dec 28 & 2MASS 0122-2439 A & Keck-I/HIRES & GG475 & $1 \times 90$ \\
\hline
\end{tabular}


TABLE 3

Properties of the 2MASS J01225093-2439505 AB System

\begin{tabular}{|c|c|c|}
\hline Parameter & Primary & Secondary \\
\hline \multicolumn{3}{|c|}{ Physical Properties } \\
\hline Age (Myr) & \multicolumn{2}{|c|}{$120 \pm 10^{\mathrm{a}}$} \\
\hline$d_{\text {phot }}(\mathrm{pc})$ & $36 \pm 4^{\mathrm{b}}$ & $\cdots$ \\
\hline$\mu_{\alpha} \cos \delta(\mathrm{mas} / \mathrm{yr})$ & $120.2 \pm 1.9^{\mathrm{c}}$ & $\cdots$ \\
\hline$\mu_{\delta}(\mathrm{mas} / \mathrm{yr})$ & $-120.3 \pm 1.7^{\mathrm{c}}$ & $\cdots$ \\
\hline$R V(\mathrm{~km} / \mathrm{s})$ & $9.6 \pm 0.7$ & $\cdots$ \\
\hline Proj. Sep. (AU) & \multicolumn{2}{|c|}{$52 \pm 6$} \\
\hline$U(\mathrm{~km} / \mathrm{s})$ & \multicolumn{2}{|c|}{$-5.4 \pm 0.6$} \\
\hline$V(\mathrm{~km} / \mathrm{s})$ & \multicolumn{2}{|c|}{$-29 \pm 3$} \\
\hline$W(\mathrm{~km} / \mathrm{s})$ & \multicolumn{2}{|c|}{$-8.0 \pm 0.7$} \\
\hline$X(\mathrm{pc})$ & \multicolumn{2}{|c|}{$-4.5 \pm 0.5$} \\
\hline$Y(\mathrm{pc})$ & \multicolumn{2}{|c|}{$-1.25 \pm 0.14$} \\
\hline$Z(\mathrm{pc})$ & \multicolumn{2}{|c|}{$-36 \pm 4$} \\
\hline $\log \left(L_{\mathrm{X}} / L_{\mathrm{Bol}}\right)$ & \multicolumn{2}{|c|}{$-3.17 \pm 0.3$} \\
\hline $\log \left(L_{\mathrm{Bol}} / L_{\odot}\right)$ & $-1.72 \pm 0.11$ & $-4.19 \pm 0.10$ \\
\hline Spectral Type & M3.5 $\pm 0.5^{\mathrm{d}}$ & $\mathrm{L} 4-\mathrm{L} 6$ \\
\hline Mass & $0.40 \pm 0.05 M_{\odot}^{\mathrm{a}}$ & $12-14$ or $23-27 M_{\text {Jup }}$ a, e \\
\hline \multicolumn{3}{|c|}{ Photometry } \\
\hline $\bar{V}(\mathrm{mag})$ & $14.24 \pm 0.07^{\mathrm{c}}$ & $\cdots$ \\
\hline$R$ (mag) & $13.2^{\mathrm{f}}$ & $\cdots$ \\
\hline$I$ (mag) & $11.3^{\mathrm{f}}$ & $\ldots$ \\
\hline$J_{\mathrm{MKO}}(\mathrm{mag})$ & $10.02 \pm 0.03^{\mathrm{g}}$ & $16.81 \pm 0.14$ \\
\hline$H_{\mathrm{MKO}}(\mathrm{mag})$ & $9.47 \pm 0.02^{\mathrm{g}}$ & $15.65 \pm 0.04$ \\
\hline$K_{\mathrm{MKO}}(\mathrm{mag})$ & $9.17 \pm 0.03^{\mathrm{g}}$ & $14.53 \pm 0.05$ \\
\hline$L^{\prime}$ (mag) & $9.0 \pm 0.1^{\mathrm{h}}$ & $13.2 \pm 0.1$ \\
\hline$M_{J}(\mathrm{mag})^{\mathrm{i}}$ & $7.2 \pm 0.2$ & $14.0 \pm 0.3$ \\
\hline$M_{H}(\mathrm{mag})^{\mathrm{i}}$ & $6.7 \pm 0.2$ & $12.9 \pm 0.2$ \\
\hline$M_{K}(\mathrm{mag})^{\mathrm{i}}$ & $6.4 \pm 0.2$ & $11.7 \pm 0.3$ \\
\hline$M_{L^{\prime}}(\mathrm{mag})^{\mathrm{i}}$ & $6.2 \pm 0.3$ & $10.4 \pm 0.3$ \\
\hline$(J-H)_{\mathrm{MKO}}(\mathrm{mag})$ & $0.55 \pm 0.04$ & $1.16 \pm 0.15$ \\
\hline$(H-K)_{\mathrm{MKO}}(\mathrm{mag})$ & $0.30 \pm 0.04$ & $1.12 \pm 0.06$ \\
\hline$(J-K)_{\mathrm{MKO}}(\mathrm{mag})$ & $0.85 \pm 0.04$ & $2.28 \pm 0.15$ \\
\hline$K_{\mathrm{MKO}}-L^{\prime}(\mathrm{mag})$ & $0.30 \pm 0.10$ & $1.33 \pm 0.11$ \\
\hline$W I S E W 1$ (mag) & $9.01 \pm 0.03^{\mathrm{j}}$ & $\ldots$ \\
\hline$W I S E W 2(\mathrm{mag})$ & $8.84 \pm 0.02^{\mathrm{j}}$ & $\cdots$ \\
\hline$W I S E W 3$ (mag) & $8.75 \pm 0.03^{j}$ & $\cdots$ \\
\hline$W I S E W 4$ (mag) & $8.2 \pm 0.2^{\mathrm{j}}$ & $\cdots$ \\
\hline GALEX NUV (mag) & $20.67 \pm 0.14^{\mathrm{k}}$ & $\cdots$ \\
\hline GALEX FUV (mag) & $21.2 \pm 0.3^{\mathrm{k}}$ & $\cdots$ \\
\hline$R O S A T$ flux $\left(\mathrm{erg} \mathrm{sec}-1 \mathrm{~cm}^{-2}\right)$ & $3.4 \pm 1.6 \times 10^{-13}$ & $\cdots$ \\
\hline
\end{tabular}

Note. - $U V W X Y Z$ values and absolute magnitudes are based on the photometric distance estimate. $U$ and $X$ are positive toward the Galactic center, $V$ and $Y$ are positive toward the direction of galactic rotation, and $W$ and $Z$ are positive toward the North Galactic Pole.

a Assumes membership to AB Dor YMG (120 Myr; Luhman et al. 2005; Barenfeld et al. 2013), otherwise a wider range of 10-120 Myr is possible.

b Computed using the Pleiades $M_{V}$ vs. $V-K$ relation from Section 3.1

c From the UCAC4 catalog (Zacharias et al. 2013). Systematic errors in proper motions are estimated to be $\sim 1-4$ mas $_{\mathrm{yr}^{-1}}$.

d From Riaz et al. (2006).

e Hot start evolutionary models overlap near the deuterium-burning limit, resulting in dual-valued mass predictions (Section 3.4).

${ }^{f}$ From the USNO-B1.0 catalog (Monet et al. 2003).

g Converted to the MKO system from 2MASS (Skrutskie et al. 2006) using the transformation in Leggett et al. (2006).

h Assumes a $K-L^{\prime}$ color of $0.2 \pm 0.1 \mathrm{mag}$ (Golimowski et al. 2004).

i Based on our photometric distance estimate.

j From the WISE All-Sky Data Release (Cutri et al.2012).

k From the GALEX GR6/7 data release (Morrissey et al. 2007). 


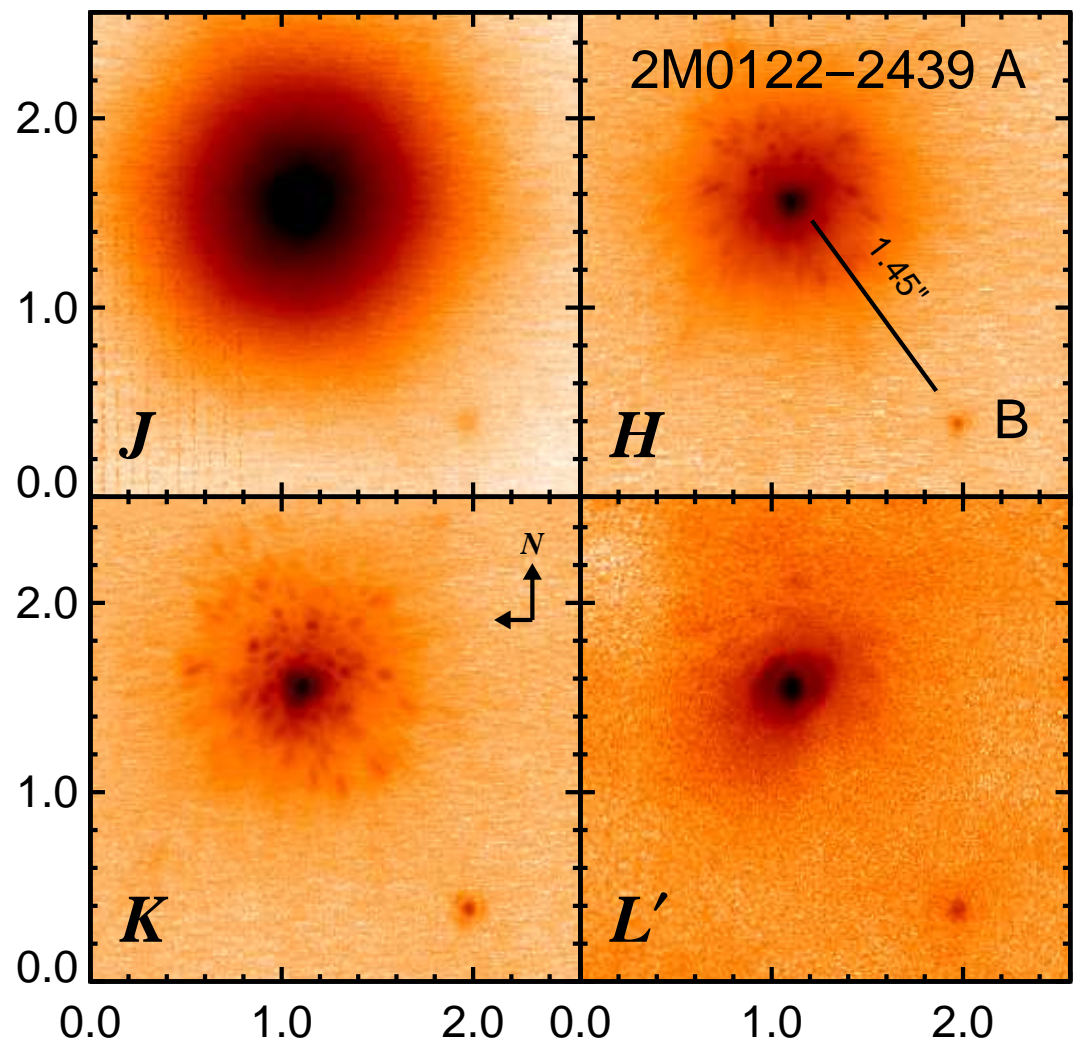

FIG. 1.- Keck/NIRC2 adaptive optics images of 2MASS 0122-2439 AB. The companion is located at a projected separation of $1.45^{\prime \prime}$ with a flux ratio of $\approx 6.2 \mathrm{mag}$ in $H$. The $J$-band data are from June 2013 , and the $H, K$, and $L^{\prime}$ images are from January 2013 . North is up and East is to the left. 


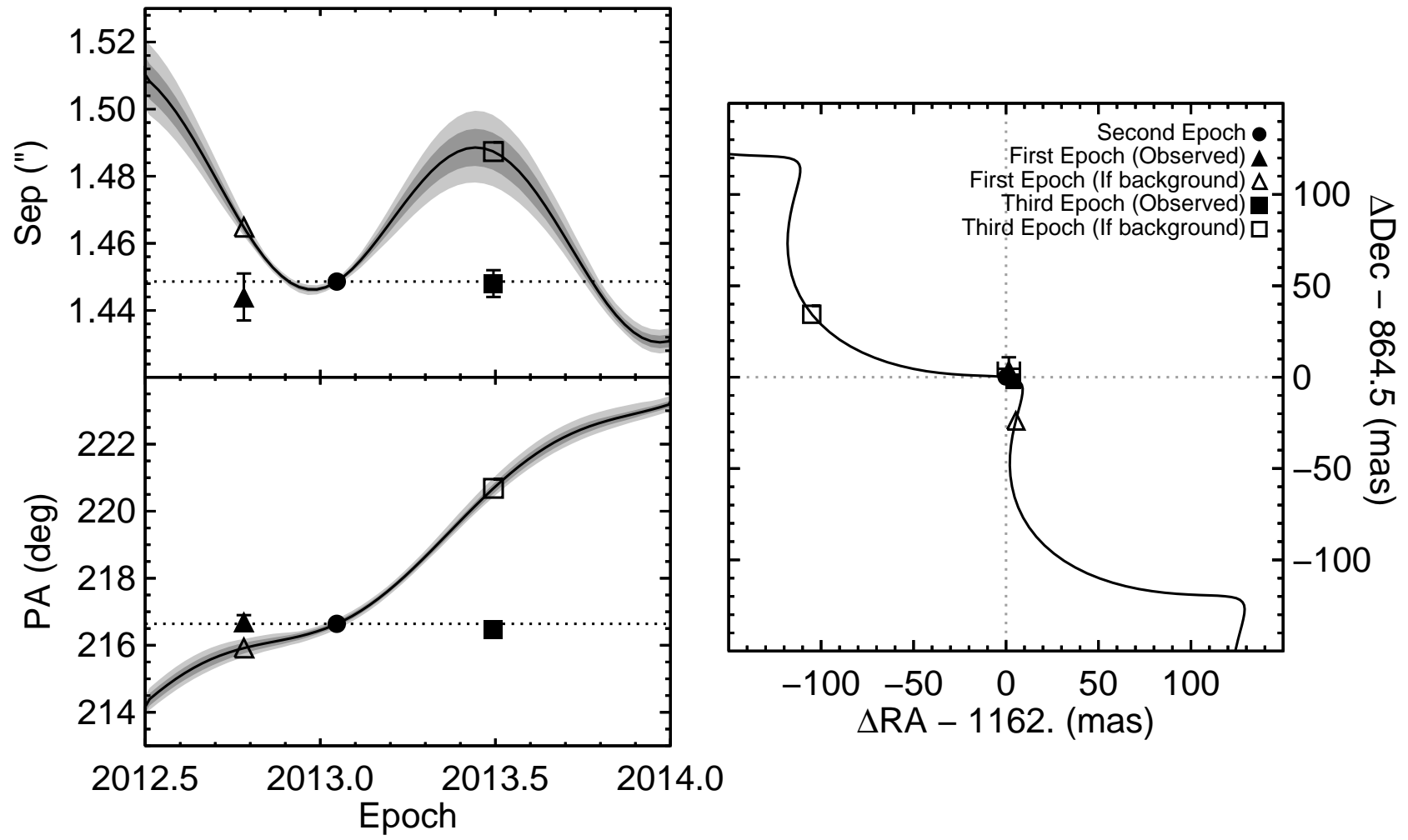

FIG. 2.- Astrometric test of common proper motion for 2MASS 0122-2439 AB. Black curves show the expected behavior of a stationary background object based on our January 2013 NIRC2 measurement for 2MASS 0122-2439 B (filled circle), the primary's proper motion, and the photometric distance (Table 3). Together our October 2012 IRCS astrometry (filled triangle) and June 2013 NIRC2 astrometry differ from the stationary track in separation (upper left) and position angle (lower left) by $28-\sigma$. The right panel shows the relative change in right ascension and declination among the three epochs. Here $\Delta$ refers to primary minus secondary position. 

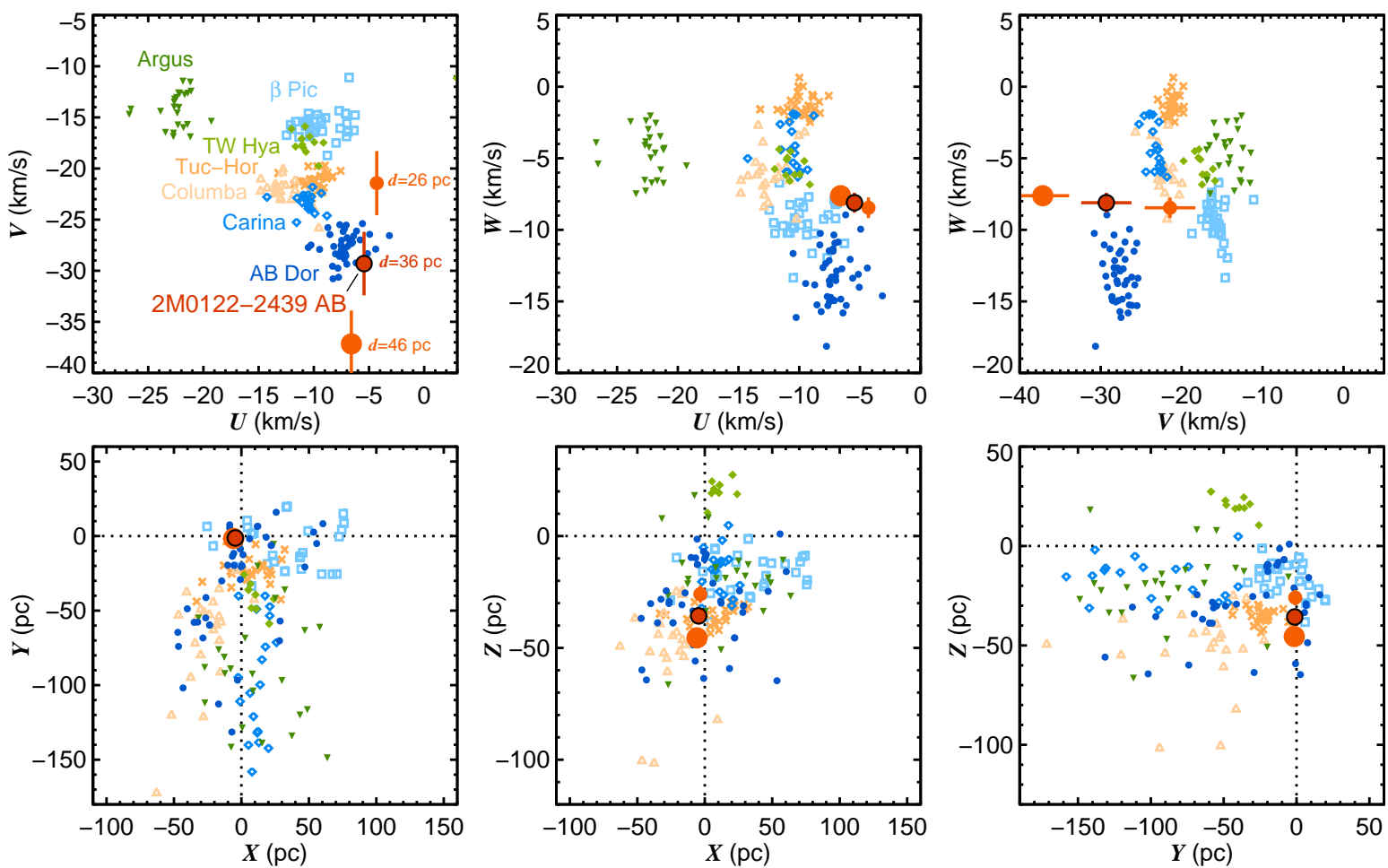

FIG. 3.- $U V W$ kinematics and $X Y Z$ space positions for 2MASS 0122-2439 AB relative to young moving groups in the solar neighborhood from Torres et al. (2008). 2MASS 0122-2439 AB is consistent with the AB Dor moving group ( $\approx 120$ Myr) in $U$ and $V$ but only marginally consistent with known members in $W$. We tentatively associate 2MASS 0122-2439 AB with AB Dor, but a parallax is needed for verification. The red circle shows our photometric distance of $36 \pm 4 \mathrm{pc}$, and the two orange circles show distances of 26 and 46 pc for comparison. Errors in kinematics and space positions for 2MASS 0122-2439 AB incorporate uncertainties in the proper motion, radial velocity, and photometric distance. 


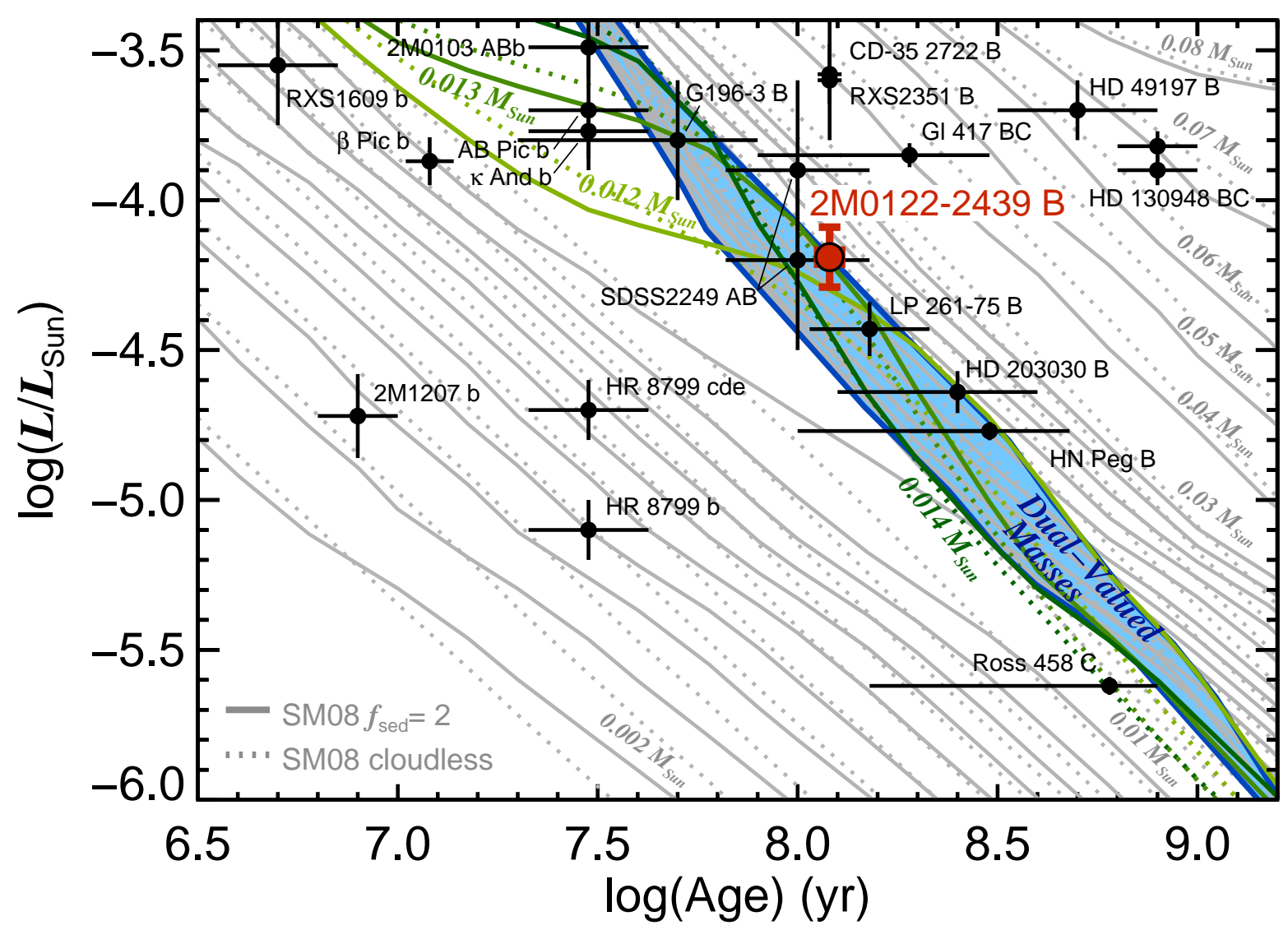

FIG. 4. - Luminosity and age of 2MASS 0122-2439 B compared with hot start cooling tracks for cloudy $\left(f_{\text {sed }}=2\right.$, solid gray curves $)$ and clear (dotted gray curves) atmospheres from the evolutionary models of Saumon \& Marlev (2008). 2MASS 0122-2439 B and several published objects fall in the region near the deuterium-burning limit where cooling tracks overlap as a result of mass-dependent deuterium-burning timescales (blue). 2MASS $0122-2439$ B is consistent with both $\approx 13 M_{\text {Jup }}$ and $\approx 25 M_{\text {Jup }}$ model tracks. Overplotted are the masses and ages of other young $(<1 \mathrm{Gyr})$ substellar companions. For most objects, ages and luminosities are compiled from the literature (Lafrenière et al. 2010; Marois et al. 2008; |Marois et al.|2010; Bonnefov et al.|2010; Bonnefov et al. |2013; | Mohanty et al.|2007; |Metchev \& Hillenbrand 2006; Luhman et al. |2007; Burgasser et al.|2010; Wahhaj et al.|2011; Bowler et al.|2012a; (Allers et al.|2010; (Dupuy et al. |2010) or measured in this work (LP 261-75 B). For the rest (2M0103-5515 ABb, $\kappa$ And b, G196-3 B, HD 49197 B, Gl 417 BC), luminosities are computed using distance estimates, $H$-band magnitudes, spectral types, and bolometric corrections using the Liu et al. (2010) relations (Delorme et al. 2013; Carson et al. 2013; Osorio et al. 2010; Reid \& Walkowicz 2006; Metchev \& Hillenbrand 2004; Kirkpatrick et al.,2001). 


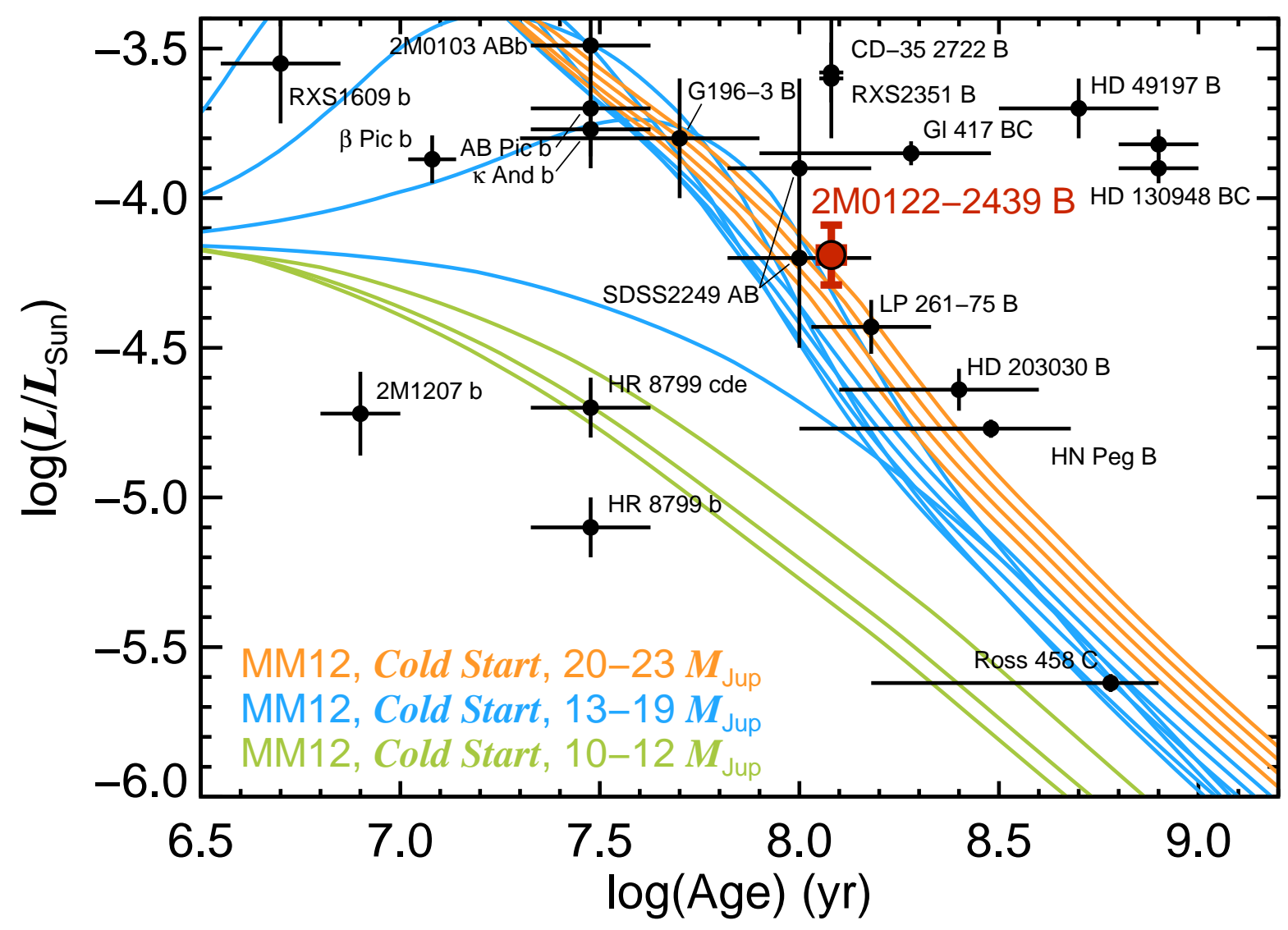

FIG. 5.- The luminosity and age of 2MASS 0122-2439 B and other directly imaged companions compared with the cold start evolutionary models from Mollière \& Mordasini (2012). For masses above $\approx 13 M_{\text {Jup }}$, deuterium burning temporarily increases the luminosity of objects formed by core accretion. Like the predictions from the hot start models (Figure 4 ), the cold start models imply masses of $\approx 14 M_{\mathrm{Jup}}$ and $\approx 23 M_{\text {Jup }}$ for 2 MASS $0122-2439$ B. Here the orange, blue, and green curves represent 10-12 $M_{\text {Jup }}, 13-19 M_{\text {Jup }}$, and $20-23 M_{\text {Jup }}$ cooling tracks in $1 M_{\text {Jup }}$ increments. See Figure 4 for details about the other companions. 


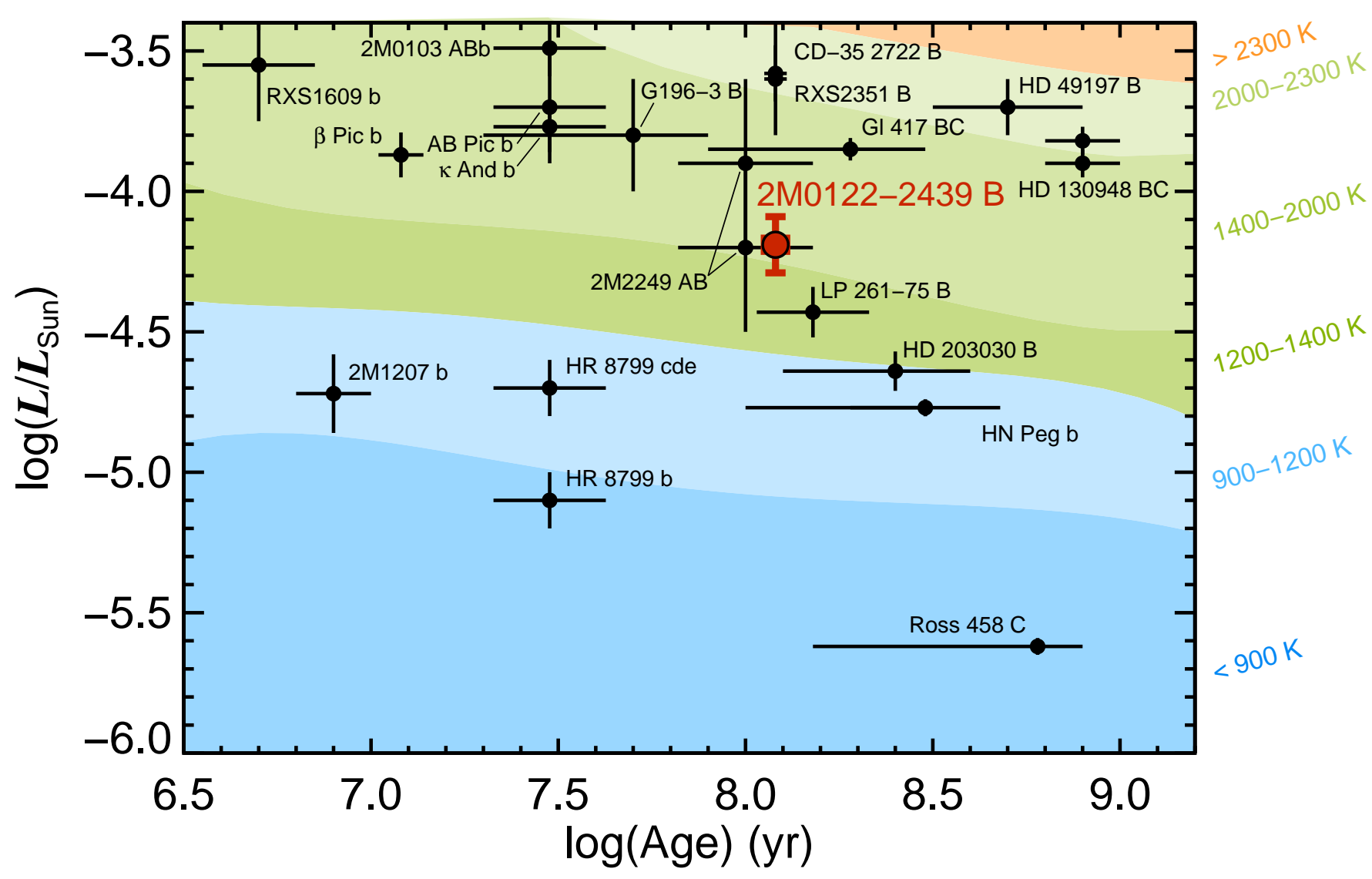

FIG. 6.- Effective temperature predictions from the Saumon \& Marlev (2008) cooling models. Colors delineate temperature regimes corresponding to the following (approximate) spectral classifications for field objects from Golimowski et al. (2004): $<$ M9 (>2300 K), L0-L3 (2000-2300 K), L3-L7 (1400-2000 K), L8-T2 (1200-1400 K), T2-T7 (900-1200 K), T8 (<900 K). Young substellar companions are overplotted for comparison (see Figure 4 for details). The predicted effective temperature for 2MASS 0122-2439 B (1350-1500 K) corresponds to the L/T transition for field objects, but we observe a mid-L spectral type. Like the HR 8799 planets, 2M1207-3932 b, and several other low-gravity L and T dwarfs, 2MASS 0122-2439 B provides further evidence that the L/T transition occurs at lower temperatures for low surface gravities. 


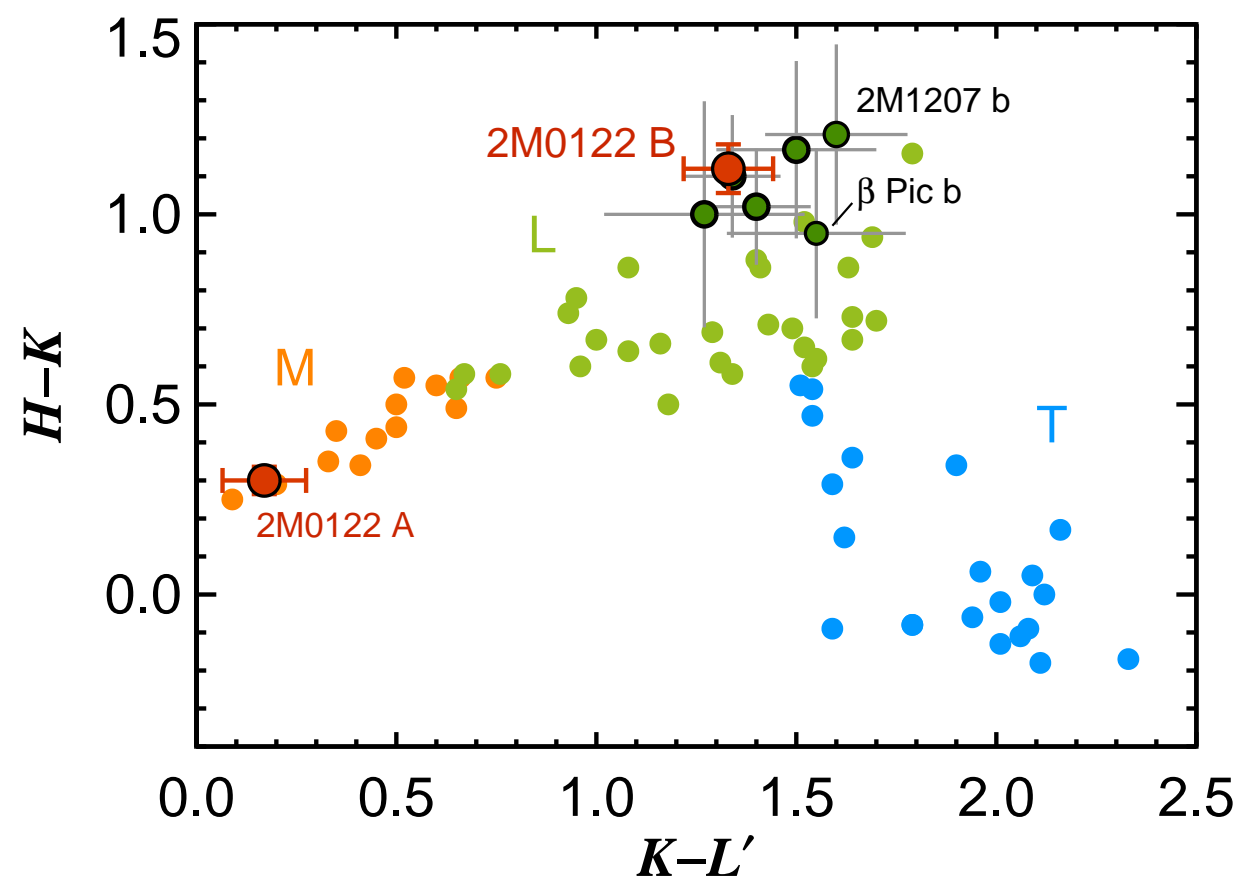

FIG. 7.- Color-color diagram showing the position of 2MASS 0122-2439 A and B relative to M (orange), L, (light green) and T (blue) dwarfs in the field. 2MASS $0122-2439 \mathrm{~B}$ is particularly red in $H-K$ compared to field objects, better resembling the planetary-mass companions HR 8799 bcde, $\beta$ Pic b, and 2MASS 1207-3932 b (green circles). Photometry for field objects is from [Leggett et al. (2010), and photometry for the planetary-mass companions is from Skemer et al. (2012), Bonnefov et al. (2013), and Mohanty et al. (2007). All photometry is in the MKO filter system. 


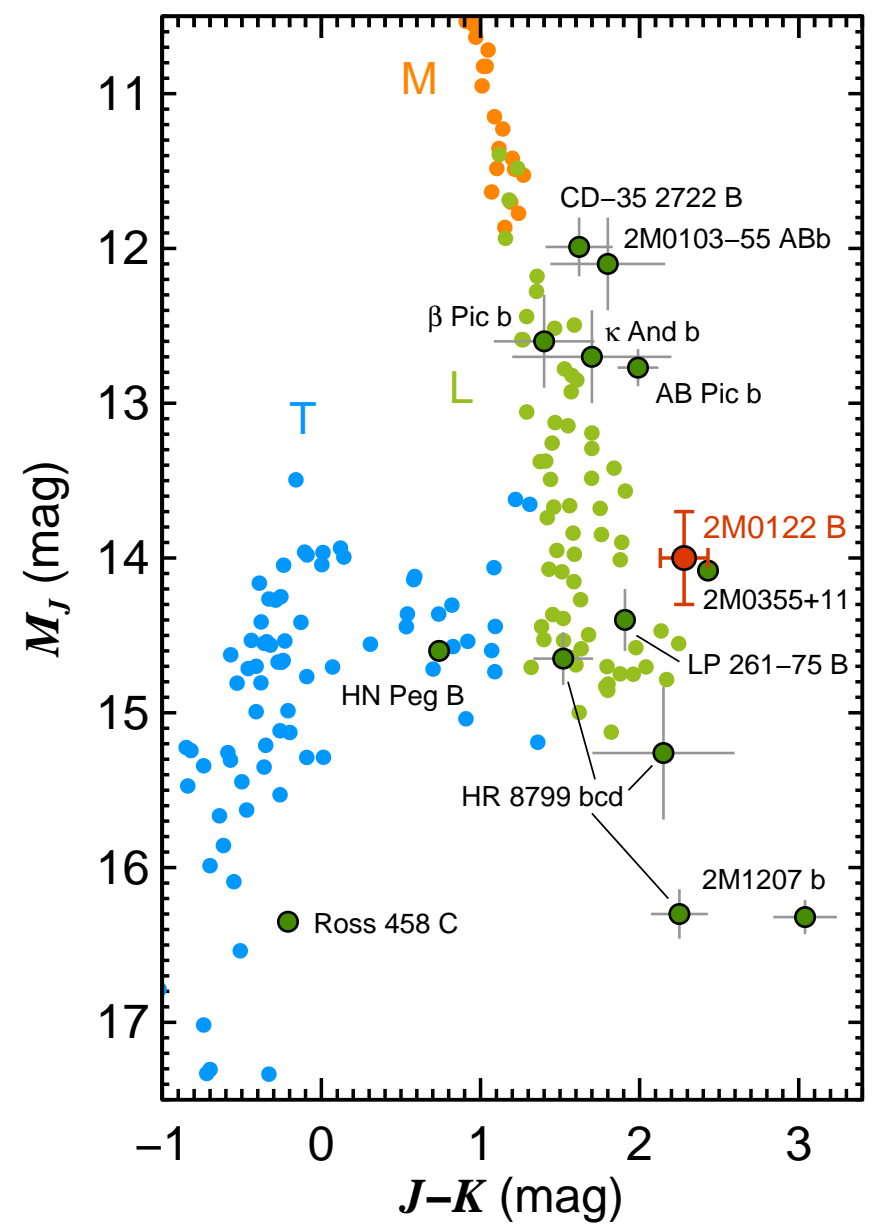

Fig. 8.- $M_{J}$ vs. $J-K$ diagram for field M (orange), L (light green), and T (blue) dwarfs. Dark green circles show substellar companions with parallactic distances (either to the primaries or the secondaries themselves). For comparison we also overplot the young, dusty L5 member of the AB Dor YMG 2MASS 0355+1133 (Cruz et al. 2009; Faherty et al. 2012; [Liu et al. 2013). The $M_{J}$ magnitude and $J-K$ color of 2MASS 0122-2439 B is similar to 2MASS 0355+1133. Photometry is from Dupuv \& Liu (2012), Skemer et al. (2012), Carson et al. (2013), Delorme et al. (2013), and Bonnefov et al. (2013). All photometry is in the MKO filter system. 

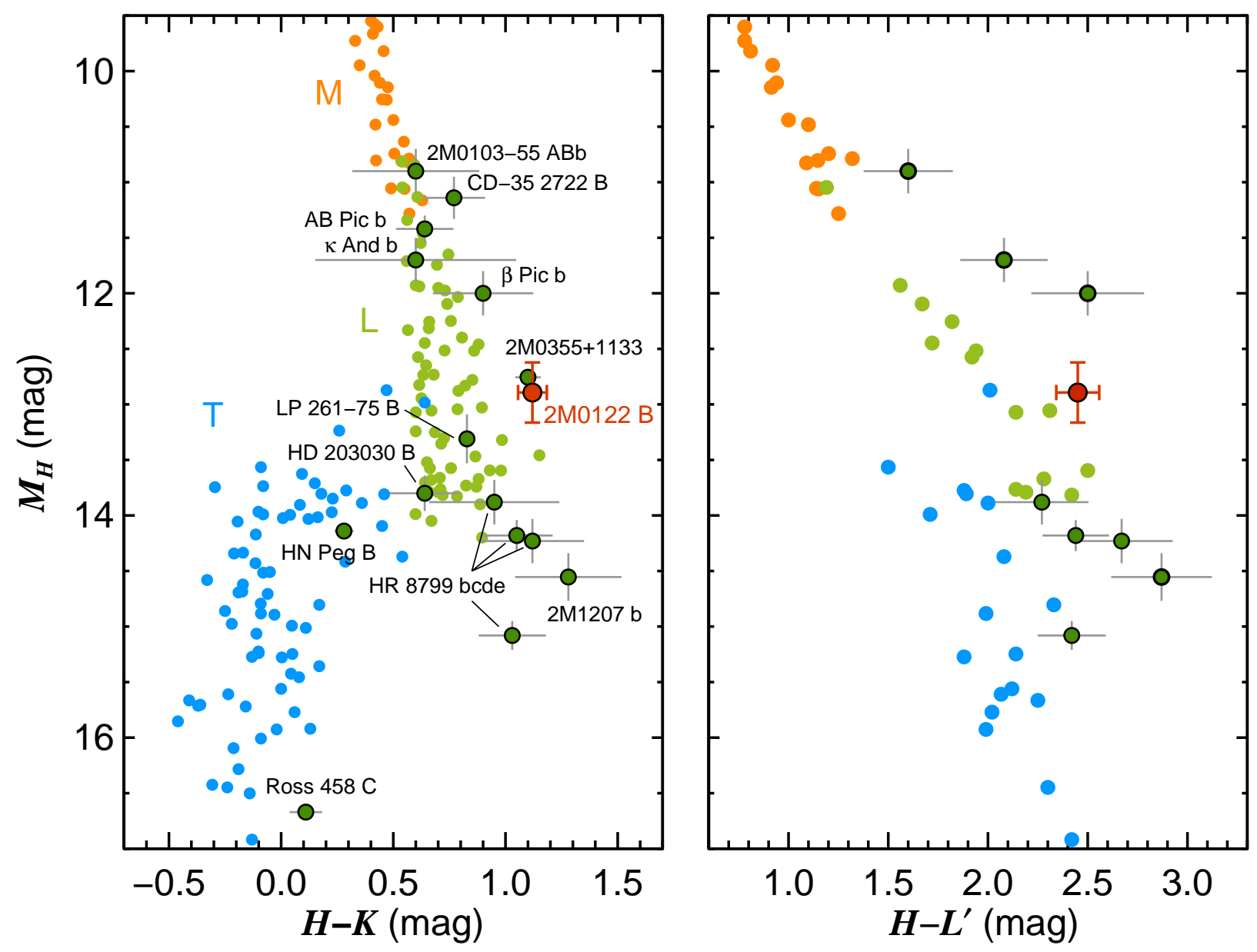

Fig. 9. - Similar to Figure 8 but for $M_{H}$ vs. $H-K$ (left) and $M_{H}$ vs. $H-L^{\prime}$ (right). 2MASS $0122-2439$ B is redder in both $H-K$ and $H-L^{\prime}$ compared to the field sequence, indicative of a young and/or dusty atmosphere (e.g., Liu et al. 2013). Photometry is from Metchev \& Hillenbrand (2006), Dupuy \& Liu (2012), Skemer et al. (2012), Carson et al. (2013), Delorme et al. (2013), and Bonnefoy et al. (2013). All photometry is in the MKO filter system. 


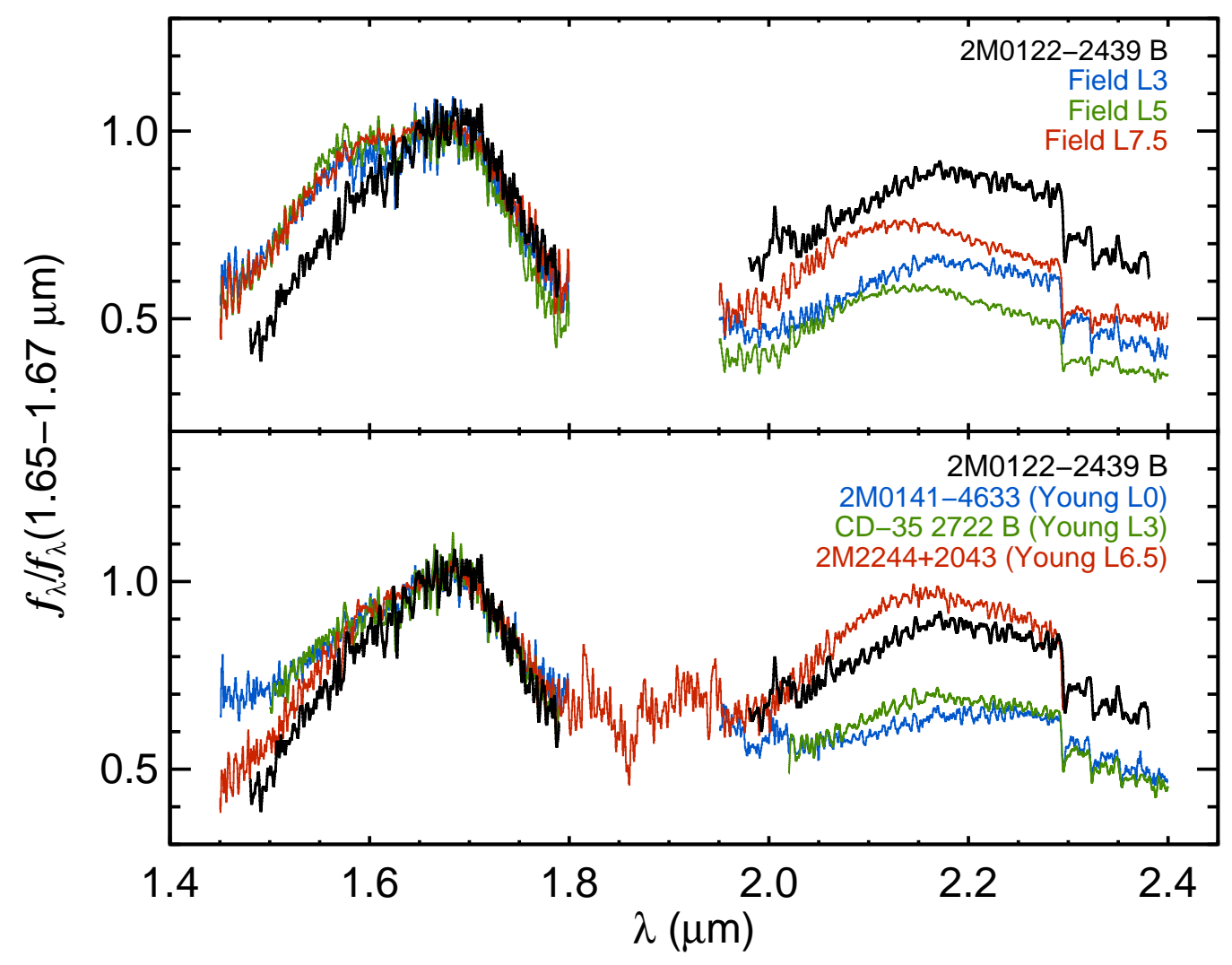

FIG. 10.- Comparison of 2MASS 0122-2439 B with field (top) and young (bottom) L dwarfs. Field objects show prominent FeH absorption between 1.57-1.62 $\mu \mathrm{m}$ and a wider $H$-band shape. 2MASS 0122-2439 B better resembles young (30-120 Myr) L dwarfs, which exhibit more angular $H$-band shapes as a result of gravity-sensitive steam absorption and collision-induced $H_{2}$ absorption (Barman et al. 2011a). 2MASS 0122-2439 B is redder in $H-K$ than the low-gravity L0 field object 2MASS J01415823-4633574 and the L3 AB Dor member CD-35 $2722 \mathrm{~B}(\sim 120 \mathrm{Myr})$, but slightly bluer than the low-gravity L6.5 object 2M2244+2043. The field spectra of 2MASS J1506+1321 (L3), SDSS J0539-0059 (L5), and 2MASS J0825+2115 (L7.5) are from the IRTF Spectral Library (Cushing et al. 2005). The young comparison objects are from Kirkpatrick et al. (2006), Wahhaj et al. (2011), and McLean et al. (2003). All spectra have been smoothed to $R \sim 1000$ and normalized between $1.65-1.67 \mu \mathrm{m}$. 


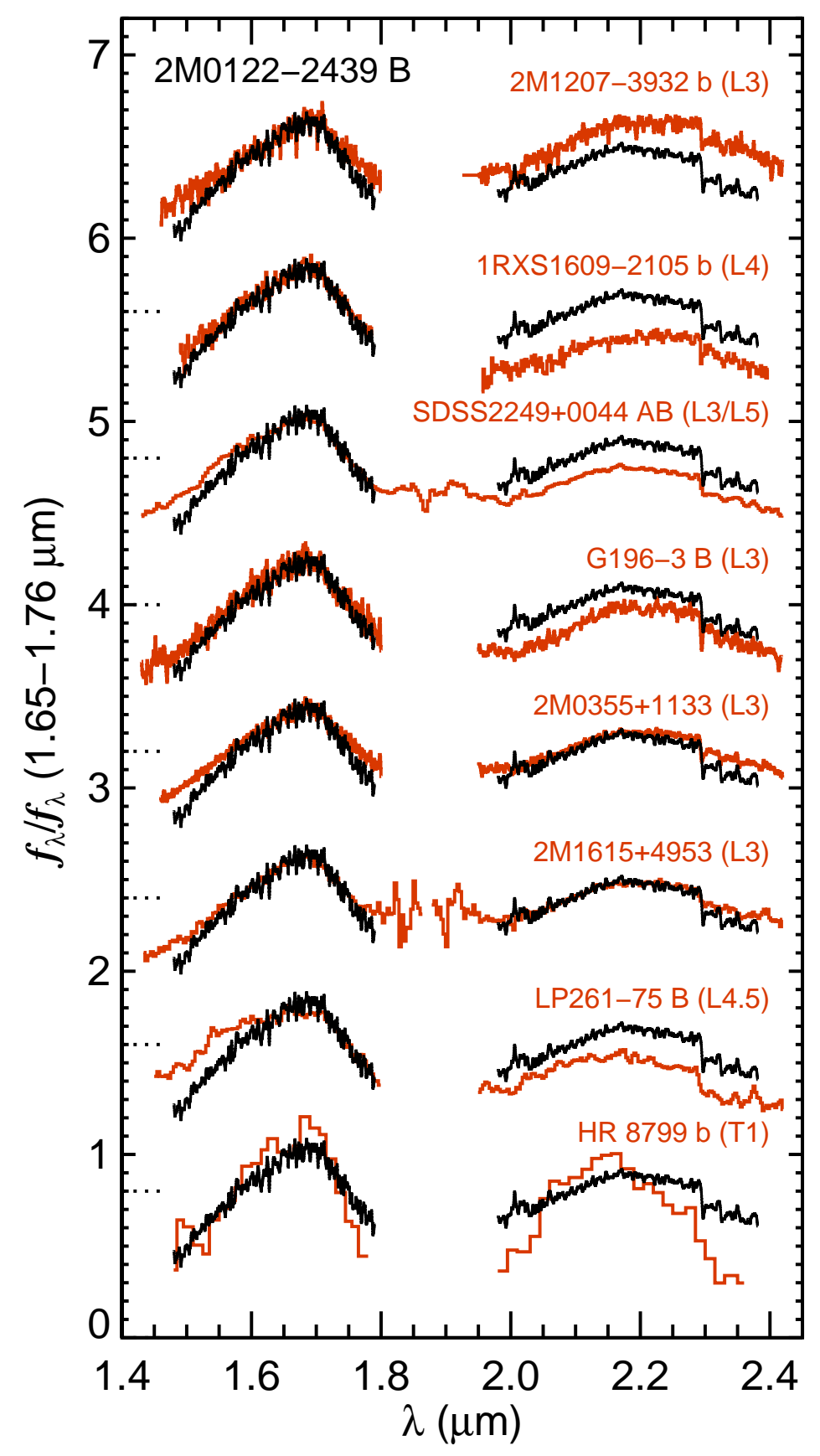

FIG. 11.- Our OSIRIS spectra of 2MASS 0122-2439 B compared with low-gravity L dwarfs. The $H$-band shape best resembles the young L4 companion 1RXS 160929.1-210524 b, the young 20-85 Myr L3 companion G196-3 B, the young field L3 dwarf 2MASS 1615+4953, and the $\approx 30 \mathrm{Myr} \sim \mathrm{T} 1$ planet HR $8799 \mathrm{~b}$. Although it has similar colors to the AB Dor member 2MASS 0355+1133, the $H$-band shape of 2MASS 0122-2439 B appears more peaked, suggesting a younger age or lower temperature for 2MASS 0122-2439 B. Several companions have low-gravity classifications in Allers \& Liu (2013) based on their spectral typing scheme: SDSS 2249+0044 AB is listed as intermediate gravity, G196-3 B is very low-gravity, 2MASS $0355+1133$ is very low-gravity, and 2MASS 1615+4953 is very low-gravity. Note that spectral classifications listed in the figure are near-infrared spectral types. Spectra are from Patience et al. (2010), Lafrenière et al. (2008), Allers et al. (2010), Allers \& Liu (2013), and Barman et al. (2011a). 


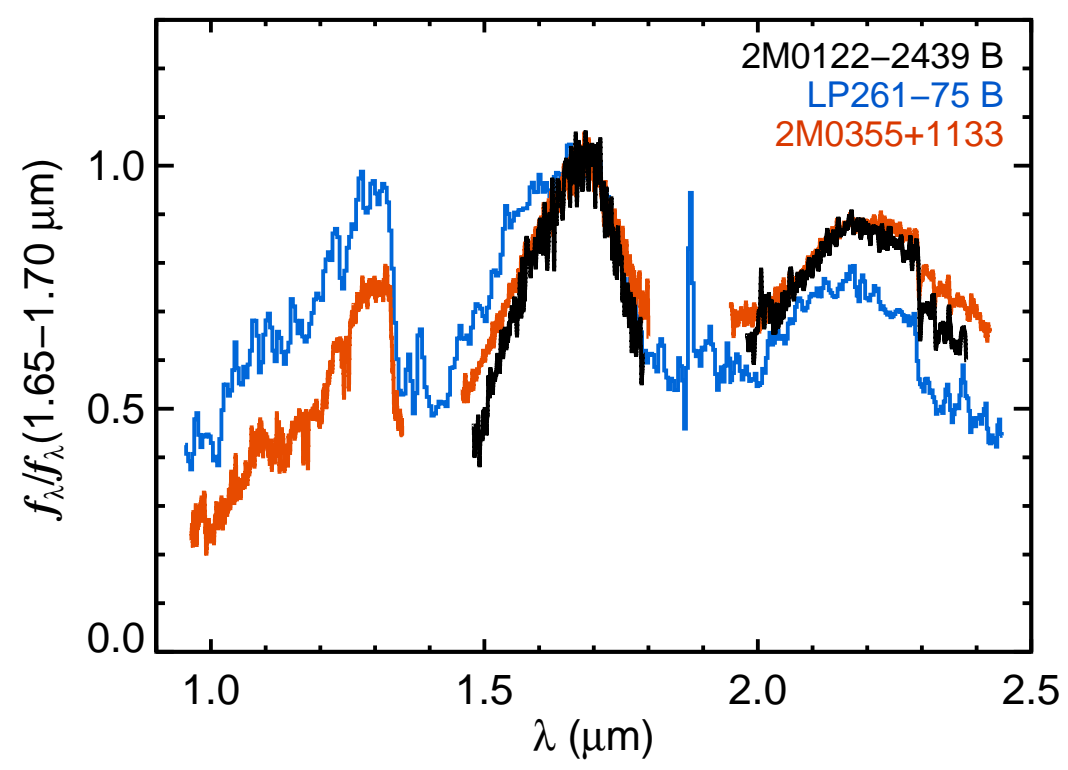

FIG. 12.- Comparison of 2MASS 0122-2439 B with the young companion LP 261-75 B and the L5 AB Dor member $2 \mathrm{MASS} 0355+1133$. 2MASS 0122-2439 B and LP 261-75 B have similar ages and luminosities, but their spectra are very different. The angular $H$-band shape of 2MASS 0122-2439 B indicates it has a lower surface gravity, pointing to a younger age and/or lower mass. The $H$-band shape is closer to (but slightly narrower than) that of 2MASS $0355+1133$, but the depth of the $2.3 \mu \mathrm{m}$ CO feature is much greater in $2 \mathrm{MASS} 0122-2439 \mathrm{~B}$.

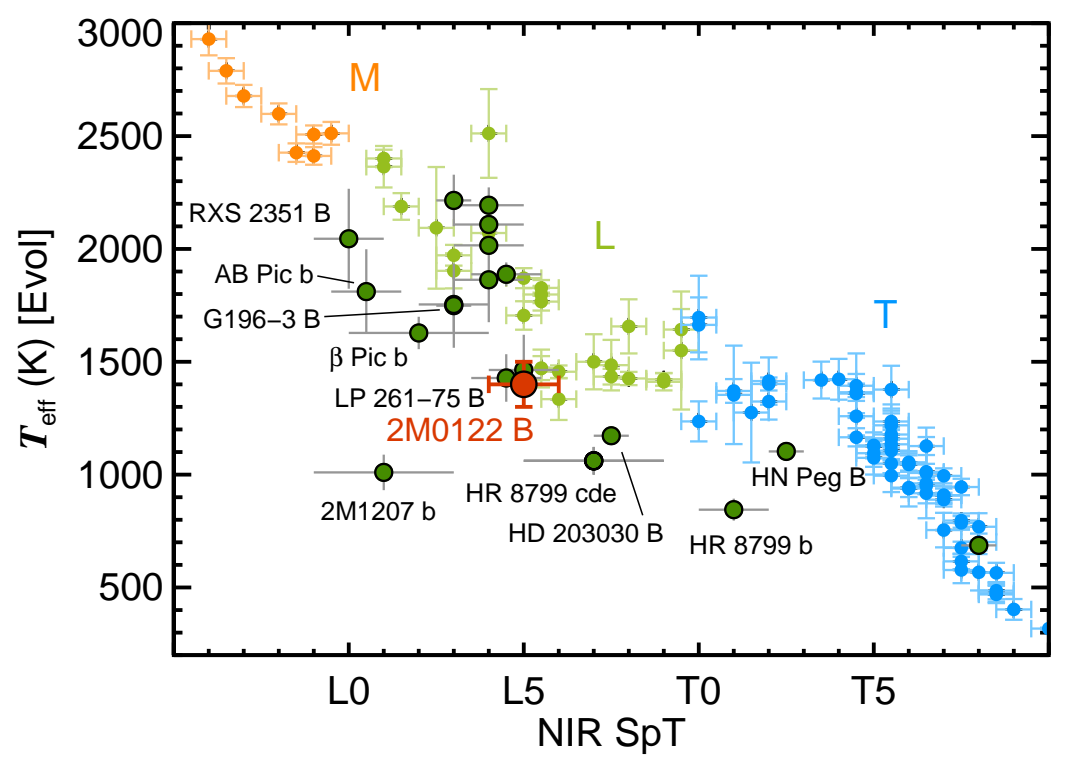

FIG. 13. - Near-infrared spectral types of old $(>1$ Gyr) field objects and young ( $<1$ Gyr) companions (green) compared to their predicted effective temperatures from the Burrows et al. (1997) evolutionary models based on their ages and luminosities. Field objects are from the Dupuv \& Liu (2012) compilation and include only single objects with normal spectral properties and measured parallaxes. Luminosities are computed using the $H$-band bolometric correction from Liu et al. (2010), and ages of $5 \pm 2$ Gyr are assumed when deriving temperatures based on an interpolated grid of evolutionary models. Ages and luminosities of the young companions are the same as in Figure 4 except here we only include objects with classifications based on near-infrared spectroscopy. Note that for some objects (e.g., 2MASS 1207-3932 b) we have made use of updated near-infrared spectral types from Allers \& Liu (2013). Young companions with spectral types offset from the field population are labeled; the remaining objects (in order of decreasing spectral type) are CD-35 2722 B (L3), SDSS 2249+0044 A (L3), 1RXS 160929.1-210524 b (L4), HD 49197 B (L4), HD 130958 BC (L4), Gl 417 BC (L4.5), SDSS 2249+0044 B (L5), and Ross 458 C (T8). 